\title{
Explosives and warfare agents remote Raman detection on realistic background samples
}

\author{
Luca M. L. Cantu ${ }^{\mathrm{a}}$, Emanuela C. A. Gallo \\ DLR (German Aerospace Center), Institute of Technical Physics, Im Langen Grund, 74239 Hardthausen, Germany
}

Received: 14 September 2021 / Accepted: 18 January 2022

(C) The Author(s) 2022

\begin{abstract}
Ultraviolet Raman spectroscopy measurements have been taken to detect non-uniform and self-prepared samples of both explosives and warfare agent simulants on realistic backgrounds. The main goal was to quantify the minimum requirements for an unambiguous identification in remote detection (distance $50 \mathrm{~cm}$ ) with a commercial Czerny-Turner spectrometer coupled with a ICCD camera. Different backgrounds in the categories of metals, natural, construction materials, transparent and plastics were used to simulate realistic encounters in a possible warzone scenario. The distribution of the chemicals was random in order to simulate a realistic measurement, and the exact detected amount was determined during the post-processing. Measurements show the capability of the developed Raman system to detect amount always in the range of $\mu \mathrm{g}$ range. Further data analysis will demonstrate the extension of the detection up to ng range for few cases. An analysis of the incident laser beam angle, size (diameter) and energy dependency is also discussed to demonstrate the technological limits outside the optimal detecting conditions.
\end{abstract}

\section{Introduction}

Chemical warfare agents are used worldwide to intentionally harm people. To contribute to the prevention of the use of such hazardous chemicals by terrorists or criminals, the Organization for the Prohibition of Chemical Weapons (OPCW) was established in 1997 to disarm the weapon of mass destruction within a time frame and the European implementation of the Regulation (EU) 98/2013 started to limit the use of the explosive precursors that can be easily accessible from the market. In order to prevent possible attacks, a reliable detection and identification system is needed. Usually, when handling warfare agents, those materials easily stick to the surfaces and their traces can be detected from the surrounding environment or might stick to the clothing's terrorist too [1]. However, the detection of these materials remains a challenge due to the small amount available over surfaces. Hence the requirement of a system with a very high sensitivity is mandatory. At the same time, a high specificity is necessary in order to discriminate an explosive from an interfering background or harmless substances. Therefore, obtaining a well-defined spectral signature that leads to a clear identification and a low rate of false alarm is very difficult from large molecules like explosives and their precursors; moreover, the background material might cover, absorb or distort the signal causing more uncertainty. Different types of technology should be employed depending on the type of compound and environmental conditions. In addition, it is desirable to achieve the widest possible range of detectable hazardous substances and, at the same time, to reduce the acquisition time. For these reasons, a comprehensive investigation of the detection possibilities and their optimization for the various warfare agents is mandatory [2]. Among all the available technologies [1,2], the ones with the best potential of being fast and able to detect and identify many explosive compounds and upgradable to new threats are laser-based spectroscopic methods for trace detection. In this context, the presented work is focused on short-pulse Raman spectroscopy chosen for remote detection. Raman spectroscopy was selected for its unique capability of being fast-responsive, highly sensitive, selective and able to identify different explosive compounds [3, 4], precursors [5] and chemical warfare agents [6]. As previously demonstrated by Gallo et al. [7], perhydro-1,3,5-trinitro-1,3,5-triazine (RDX) [8] was possible to be detected in the $\mu \mathrm{g}$ range at about $60 \mathrm{~cm}$ distance using well-defined homogeneous samples over an polished aluminum background. This work is a follow-up of that study. In this new development, RDX and trinitrotoluene (TNT) [9] were tested again over new NATO-237 standard samples, prepared with lower concentrations with respect to the previous study [7] and used as reference. The purpose was to detect the minimum possible explosive amount over an ideal polished surface with a homogeneous distribution of the chemical under examination. However this represents an ideal laboratory condition. Then, further background materials were chosen considering the highest probability of encountering them into a war zone (a realistic scenario). This includes different kind of scenarios such as urban areas, flat lands and forests. None of the materials were newly purchased or represent an ideal surface, but they show sign of usage, degradation dirtiness and impurities to better simulate a realistic scenario. The background materials collected were summarized in five sub-categories as construction materials, glass materials, metallic

\footnotetext{
a e-mail: lucamlc@vt.edu (corresponding author)
} 
materials, natural materials and plastic materials. Traces of RDX, TNT, Glyphosate [10] (GLP, as simulant of nerve agent Sarin) and Triphenyl-Phosphate [11] (TPP, as simulant of nerve agent VX) were deposited on realistic backgrounds, and the exact amount was then calculated after the measurements using a 3D laser microscope. The choice of the surrogates was based on their main functional group similarities (hence similar Raman signal) according to Singer et al. [12]. The thresholds and limits of selectivity and sensitivity are recorded and discussed, as well as the laser incident angle, laser beam diameter and associated laser energy dependency and the detection distance (fixed at $50 \mathrm{~cm}$ in this work) for the respective explosive, warfare agent simulant and background.

\section{Optical Setup}

A picture of the optimized remote detection Raman setup is shown in Fig. 1.

An InnoLas 2500 laser was employed to provide a laser beam at $355 \mathrm{~nm}$ wavelength at $20 \mathrm{~Hz}$ repetition rate (pulse duration $<8 \mathrm{~ns}$ ). The output laser beam was kept constant at $1.0 \mathrm{~cm}$ diameter for most of the tests. A removable telescope was placed in the middle of the laser path in order to be able to change the laser beam size with minimal effort. At the measurement position the laser energy was measured about $8 \mathrm{~mJ} /$ pulse and kept constant. The incident beam was about $10^{\circ}$ to the normal surface of the target to avoid direct reflections of the laser beam into the collecting optics, and then it was sent into the spectrometer: this arrangement prevents damaging the camera. The sample holder was equipped with movable arms in order to allow the rotational movements required to perform the angle dependency study. The laser beam was not focused to the sample in order to avoid destroying/damaging it and to increase the sampling area. A 75-mm-diameter, 200-mm FL lens was placed at $50 \mathrm{~cm}$ distance from the sample to collect the Raman. The signal was first collimated by a 100-mm FL lens and then focused by a 75-mm FL lens into the 550- $\mu \mathrm{m}$ spectrometer's slit. A 355-nm long-pass filter was placed before the focusing lens to reject the remaining laser stray light. The spectrometer (Princeton Instruments Acton SP2500) was equipped with a UV holographic grating with a dispersion of 2400 grooves/mm. The dispersed signal was then recorded by a Princeton Instruments PI-MAX4 intensified camera (ICCD, chip size $1024 \times 512$ pixels, RB-SL intensifier $28 \%$ quantum efficiency in the UV) through LightField 4.10 software. The camera was triggered by the laser pulses, and each measurement was collected using 6.5 ns gate.

Fig. 1 Remote detection Raman setup

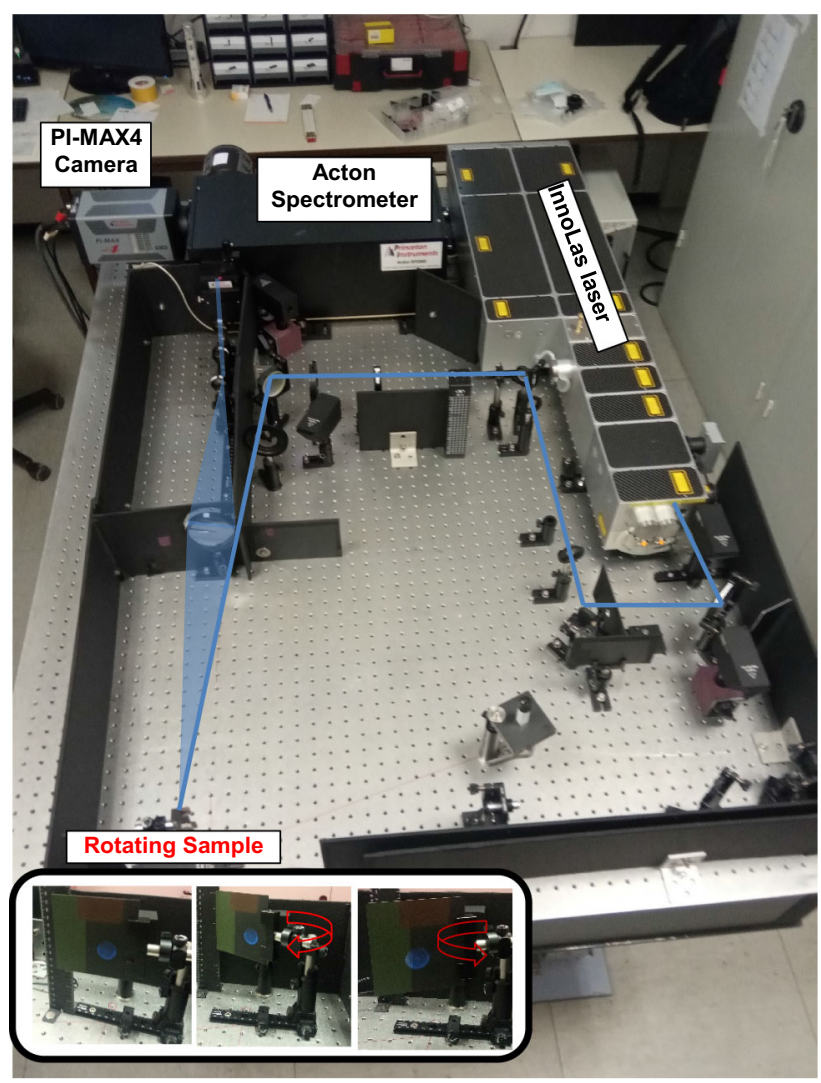


Table 1 RDX samples characteristics

\begin{tabular}{|c|c|c|c|c|c|c|}
\hline RDX Sample\# & Solvent & Concentration $[\mathrm{mg} / \mathrm{ml}]$ & Array & Droplets per point & Layers & Final distribution $\left[\mu \mathrm{g} / \mathrm{cm}^{2}\right]$ \\
\hline 1091 & $\mathrm{ACN}$ & 5 & $25 \times 20$ & 13 & 3 & 50 \\
\hline 1092 & $\mathrm{ACN}$ & 5 & $25 \times 20$ & 11 & 2 & 30 \\
\hline 1093 & $\mathrm{ACN}$ & 5 & $25 \times 20$ & 7 & 2 & 20 \\
\hline 1094 & $\mathrm{ACN}$ & 5 & $25 \times 20$ & 7 & 1 & 10 \\
\hline 1095 & $\mathrm{ACN}$ & 5 & $25 \times 20$ & 3 & 1 & 5 \\
\hline 1096 & $\mathrm{ACN}$ & 5 & $20 \times 19$ & 1 & 1 & 1 \\
\hline
\end{tabular}

Table 2 TNT samples characteristics

\begin{tabular}{|c|c|c|c|c|c|c|}
\hline TNT Sample\# & Solvent & Concentration $[\mathrm{mg} / \mathrm{ml}]$ & Array & Droplets per point & Layers & Final distribution $\left[\mu \mathrm{g} / \mathrm{cm}^{2}\right]$ \\
\hline 1111 & $\mathrm{ACN}$ & 30 & $24 \times 20$ & 5 & 1 & 50 \\
\hline 1114 & $\mathrm{ACN}$ & 30 & $24 \times 20$ & 3 & 1 & 30 \\
\hline 1115 & $\mathrm{ACN}$ & 30 & $24 \times 20$ & 2 & 1 & 20 \\
\hline 1116 & $\mathrm{ACN}$ & 30 & $24 \times 20$ & 1 & 1 & 10 \\
\hline 1117 & $\mathrm{ACN}$ & 3 & $24 \times 20$ & 5 & 1 & 5 \\
\hline 1118 & $\mathrm{ACN}$ & 3 & $24 \times 20$ & 1 & 1 & 1 \\
\hline
\end{tabular}

\section{Explosive NATO-SET-237 samples}

The laboratory samples here presented were produced as NATO-SET-237 [13] by the Fraunhofer ICT using a GeSiM 2.1 NanoPlotter ( 40-500 pico-liters per droplet) drop-on-demand inkjet printer [1]. A detailed description of this methodology is provided by Schnürer et al. [14]. The new set of samples requested was in smaller concentration comparing the previous one prepared on 2019 [7]. Smaller concentrations $\left(50,30,20,10,5\right.$ and $1 \mu \mathrm{g} / \mathrm{cm}^{2}$, respectively) were required to further test the sensitivity limits. Tables 1 and 2 summarize the characteristics of the produced samples and the different printing parameters chosen for RDX and TNT, respectively. All the samples were prepared in acetonitrile (ACN) solvent.

The critical amounts are the ones below $5 \mu \mathrm{g} / \mathrm{cm}^{2}$ : the concentration of the initial solution before printing was very low $(5 \mathrm{mg} / \mathrm{ml}$ for RDX and $3 \mathrm{mg} / \mathrm{ml}$ for TNT). Moreover, only 1 layer was printed. Therefore, the evaporation of the solvent carried some of the explosives reducing the amount deposited on the surface before it can be fully crystallized. The methodology of crystallization is quite important since the deterioration of the TNT is fast in time. As demonstrated by Yu et al. [15], TNT deteriorates more than $60 \%$ at room temperature after 20 days. This implies that the complete set of tests should be performed quite close to the time of the production of the samples. Contrary, RDX is much more stable compound with a slower deterioration time: after 40 days, less than $40 \%$ is deteriorated [15] Nevertheless, timing is quite important for RDX too; therefore, the smallest concentrations were tested immediately after the delivery of the samples. Uncertainties were expected into the final calculation since the tested amount may be smaller than the nominal value.

\section{Samples over realistic backgrounds preparation}

The realistic background materials collected can be summarized in five sub-categories: construction materials, transparent materials, metallic materials, natural materials and plastic materials. Each background was sprinkled with the pure chemical substance using the simple pressure of the finger till the compound adheres strongly to the surface. The pressure applied varied from the background type and by the chemical. For example, TNT was in very fine grains which do not stick easily so a stronger pressure was necessary. On the other hand, GLP tends to conglomerate very easily, so minimal effort was required to obtain a good adherence. Regarding the backgrounds, rough materials like asphalt brick or barks offered enough grooves for easy application of chemicals, while quartz cuvette provides some difficulties due to its polished surface. It must be also considered that all the samples, due to the optical setup configuration, must be tested vertically; this implies that each trace must sustain its own gravity. Due to the hazardousness of the chemicals involved this procedure was performed under chemical fume hood and wearing the appropriate chemical gloves protection. Pictures of selected backgrounds are shown in Fig. 2, and the white color area represents the deposited traces of TPP.

Looking at Fig. 2 some consideration has to be made. First of all, this methodology was quite straight forward and do not require the employment of any device or extra cost except for the chemicals. In addition, the distribution of the samples was purposely 


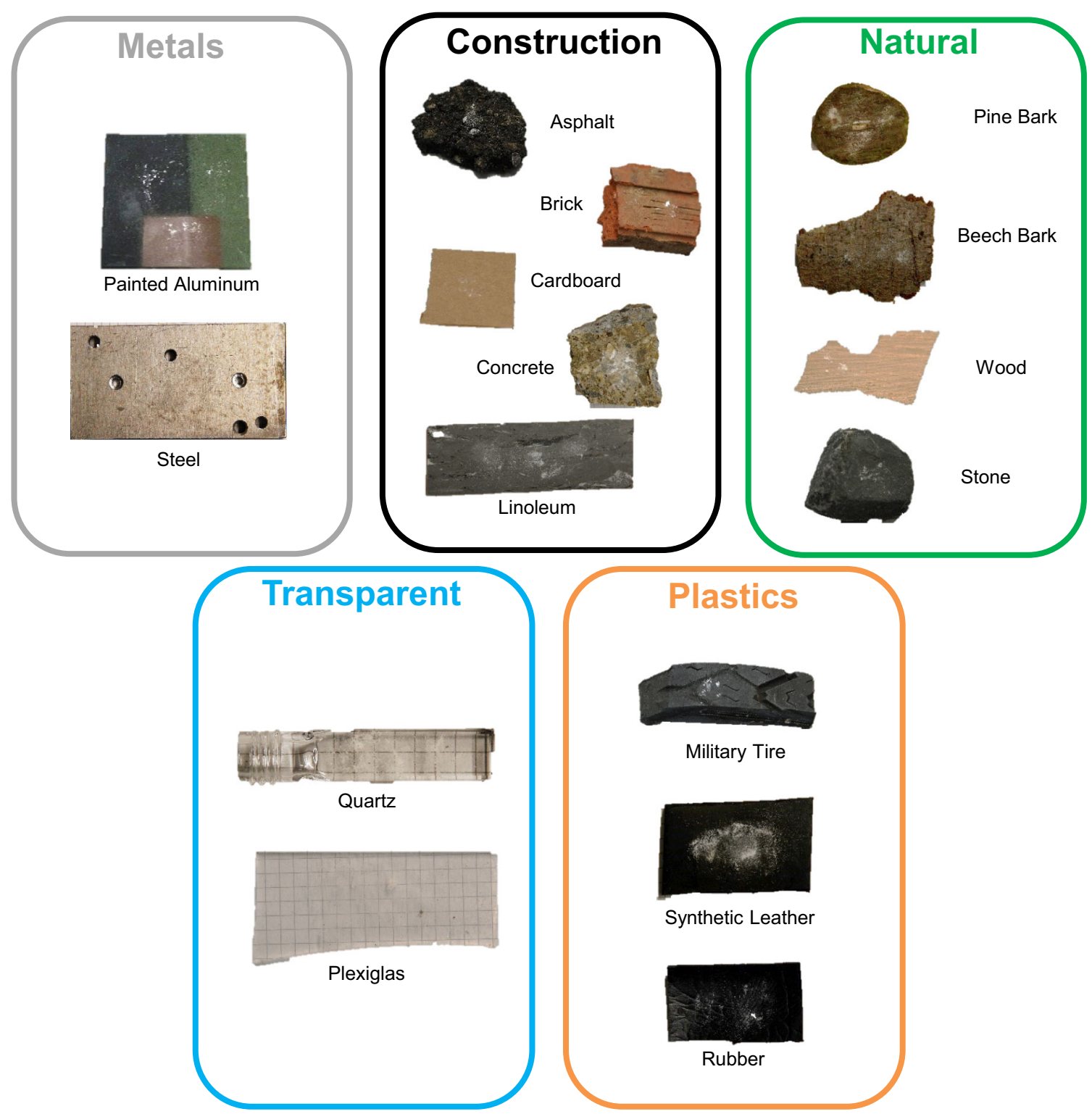

Fig. 2 TPP on realistic backgrounds

random because neither a standard amount nor a uniform distribution is expected to be found in a possible realistic warzone scenario. However, this procedure made each sample unique, so it was not possible to reproduce the same sample twice. For this reason, for each background multiple measurements were taken in order to have enough statistical meaning. Also, the amount deposited on each location was unknown at the time of the test. Controlling the chemicals amount was a very challenging task. First of all, it was not available a chemical scale with a sensitivity smaller than $1 \mathrm{mg}$. In this way it was impossible to control the amount of chemical deposited, particularly when the detection limits are desired in the $\mu \mathrm{g}$ range. Secondly, the material lost on the chemical gloves used to deposit the powder over a surface or the chemical loss because of a lack of adherence when the sample placed in vertical position was impossible not only to quantify but also to estimate. Hence, the amount of chemicals at each measurement was precisely measured later during the post-processing using a laser microscope. The procedure will be described in following Data Analysis Sect. 5. 


\section{Data analysis}

The data analysis was carried out completely after the tests. The first step was subtracting the background noise from each spectrum in order to have the clear signal to analyze. The further step was to evaluate the amount of explosive or warfare agent simulant deposited on the surface: this required a further measurement campaign involving a laser microscope.

\subsection{Background subtraction}

Different types of background noise were present in each measurement disturbing or distorting the results: camera noise, laser stray light, room light, native fluorescence of the compound and/or substrate. It is important to identify each source of noise in order to have the clearest signal possible and therefore an unambiguous identification of the possible threat.

The camera noise was minimized keeping the ICCD at a constant temperature of $-25^{\circ} \mathrm{C}$. To reduce the possible sources of signal disturbance, a camera noise background was acquired (intensifier disabled) after each measurement and automatically subtracted to the recorded spectrum.

The laser stray light was another possible source of interference. As described in the optical setup section a 355-nm long-pass filter was mounted in front of the slit of the spectrometer. This filter was useful to reject all the wavelengths lower than $355 \mathrm{~nm}$ with an efficiency of $95 \%$. Since the Raman signal is always generated at higher wavelengths, this method provided a good solution to reject most of the unwanted light inside the spectrometer.

Room light was purposely added to the measurement to provide a source of interference that simulates the day light and hence to simulate a more realistic scenario. Native fluorescence was summing up to the room light adding extra disturbances. Native fluorescence can come from the measured compounds (in this case only from the warfare agents' simulants, since it was demonstrated that none of the tested explosives offered detectable fluorescence signal [7]) and from the substrates simultaneously. The fluorescence interference can play a very significant role since the lased-induced fluorescence (LIF) signal is much stronger than the Raman [1].

To minimize this undesired effect, the best solution was to minimize the camera exposure. The gate width of the ICCD employed for this study was set at its minimum (6.5 ns): this feature cut most of the room light and the fluorescence too but not completely. It is evident that even few percentages of LIF interference can have a strong influence on the Raman signal. In this case a compromise must be found: there is no availability on the market of a detecting device to be able to have a fast gating (in the ps range) and at the same time offering a reliable quantum efficiency in the UV. So, in order to have a clear Raman signal to analyze, the solution was to remove the remaining fluorescence interference during the post-processing. Each individual spectrum was processed using OriginLab 2021: the baseline of the spectrum was mathematically interpolated with a spline curve and subtracted to the original data. Test-retest was done to verify the reliability of the data set over time. For TNT (fast evaporating-fast deteriorating under day light) similar quantities in different sampling area were taken under examination to verify the reliability of the purposed sensor, monitoring the laser energy. A fluctuation in laser energy of about $1.5 \%$ led to a signal peak change below $0.3 \%$.

\subsection{Quantification of the Traces Amount}

The quantification of the exact amount of the explosives and chemical warfare agent simulants over the realistic backgrounds was conducted after the measurements. A high-definition picture was taken after each sample preparation, and it was used to mark unambiguously the measurement location. Then all the samples were analyzed under a laser measuring microscope Olympus LEXT $O L S 40003 D$ (accuracy $\pm 2 \%$ ) with a magnification factor of 50x. Each image taken with the laser microscope was then processed using ImageJ2 $1.53 \mathrm{j}$ image software [16]. The image processing is illustrated in Fig. 3.

A threshold that was $20 \%$ of the peak value was applied to each single microscope image to force each pixel to be either black (pixel value 0 ) or white (pixel value 1). Then it was possible to calculate the percentage of the white portion of the picture. Knowing the size of the image from the microscope, $2583 \times 2574 \mu \mathrm{m}$, it was possible to calculate the area covered by the deposited chemical. The calculated area error is $\pm 4 \%$ due to the microscope accuracy. The laser microscope was also able to measure the thickness of the sample and hence calculate the total volume occupied. The overall volume error is $6 \%$. Finally, knowing the density of each compound from the literature it was possible to calculate the exact quantity for each measurement performed. A further consideration must be done. As a first approximation, only the area illuminated by excitation laser beam generates the signal. This assumption is

Fig. 3 Starting procedure for quantifying the deposited amount using ImageJ. On the left the raw image from the laser microscope (TPP on aluminum); on the right the same image after applying a black and white threshold

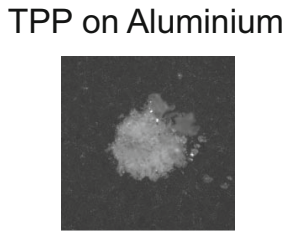

$2583 \times 2574 \mu m^{2}$
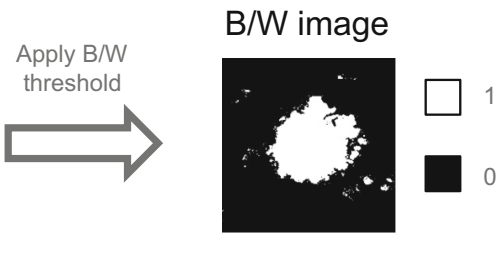
Table 3 Summary of the penetration depth values used for the tested compounds

\begin{tabular}{llll}
\hline $\mathrm{RDX}[\mu \mathrm{m}]$ & TNT $[\mu \mathrm{m}]$ & GLP $[\mu \mathrm{m}]$ & TPP $[\mu \mathrm{m}]$ \\
\hline 1.99 & 0.80 & Measured thickness & Measured thickness \\
\hline
\end{tabular}

true in case of non-resonant Raman scattering. However, in case of resonance Raman scattering (and fluorescence too) absorption effects should be taken into consideration. In case of solid-state compounds, the penetration of the laser (penetration depth) of deep UV light in many materials is very small $[17,18]$, reducing the total signal that might be generated.

For example, consider a $1 \mathrm{~cm}^{2}$ of TNT uniformly distributed over a surface with an average thickness of $1 \mathrm{~cm}$. A $355-\mathrm{nm}$ laser beam of $1 \mathrm{~cm}^{2}$ illuminated perpendicularly the TNT sample. In this case the contribution to the signal would be the $1 \mathrm{~cm}$ of the TNT plus a contribution of $0.80 \mu \mathrm{m}$ from the TNT penetration depth, according to Amin et al. [19] Hence, out of the $1.65 \mathrm{~g}$ deposited on the surface, only $132 \mu \mathrm{g}$ contributed to the signal.

The values used for the penetration depth were taken from the literature; therefore, $0.80 \mu \mathrm{m}$ was used for TNT and $1.99 \mu \mathrm{m}$ for RDX [19]. No information was found about the penetration depth of the warfare agents' simulants; therefore, the measured thickness from the laser microscope was considered to evaluate the total deposited amount. Table 3 summarizes the used values.

\subsection{Limits of detection}

In order to evaluate the sensitivity limits of a laser technique, some unique parameters must be employed. The limit of detection (LoD), defined as the lowest concentration of an analyte in a sample that can be detected, was found to be a reliable method. The method proposed by Shrivastava et al. [20], and here adopted, relies on the calculation of the signal-to-noise ratio (SNR $=2 \mathrm{H} / \mathrm{h})$. Where $\mathrm{H}$ is the height of the peak corresponding to the compound concerned and measured from the maximum of the peak to the extrapolated baseline of the signal, while $\mathrm{h}$ is the maximum fluctuation of the background noise, observed over a distance of 20 times the width at half-eight of the main peak.

When SNR is equal 3 or higher, the requirements for the LoD are met. Since all the explosives and warfare agent simulants are not simple diatomic molecules but complex ones, more than one single peak is expected. So, it was decided to use as a reference for the calculation of the SNR the strongest peak at $355 \mathrm{~nm}$ excitation wavelength. Hence, for RDX the sensitivity was calculated referring to the main $\mathrm{CN}$ stretch [21, 22] peak at $885 \mathrm{~cm}^{-1}$; for TNT the $\mathrm{CH}_{2}$ scissors band located at $1483 \mathrm{~cm}^{-1}$ was used. Several publications $[17,18,21]$ reported another strong peak at $1537 \mathrm{~cm}^{-1}$ related to the asymmetric stretching of $\mathrm{NO}_{2}$ : however, molecular oxygen $\mathrm{O}_{2}$ Q-branch [23] is located at $1554 \mathrm{~cm}^{-1}$. It must be considered that atmospheric $\mathrm{O}_{2}$ provided a very strong signal comparing TNT; therefore, it is assumed that the asymmetric stretching of $\mathrm{NO}_{2}$ was completely masked and impossible to detect. To support this statement, during the measurement campaign, the spectrometer grating was moved to the $2500 \mathrm{~cm}^{-1} \mathrm{spectral}$ region where no TNT signal was found but a peak at $2331 \mathrm{~cm}^{-1}$ corresponding to the Q-branch of $\mathrm{N}_{2}$ [23]. Other tests proved that the spectrum of air was also found in the measurements of RDX, GLP and TPP. Since GLP and TPP were employed as simulants of nerve agents, the absolute main peaks of these two compounds were not considered, but the strongest peak related to the substance they were used as substitute. For GLP the peak at $773 \mathrm{~cm}^{-1}$ (P-O-C symmetric stretch) [24] corresponded to the strongest peak of Sarin. Regarding TPP, the peak at $1226 \mathrm{~cm}^{-1}(\mathrm{P}=\mathrm{O}$ stretching) [25] was chosen as representative of the strongest peak of VX.

\section{Results}

The RDX and TNT on the NATO-SET-237 standard samples are prepared with the aim of finding the lowest possible detectable amount with the Raman system presented. Those samples are standardized samples with uniform printing distribution at low concentration levels, and they represent an ideal scenario. Explosive samples are discussed in separate chapter when applied on realistic backgrounds and their sensitivity will be discussed too. Subsequently, results and sensitivity about the warfare agent simulants will be presented individually on realistic backgrounds. Finally, a study about the influence of the incident laser angle dependence, laser beam diameter size and laser energy will be discussed.

\subsection{Explosives on NATO-SET-237 Standard Samples}

The new standard NATO-SET-237 samples of RDX and TNT were tested in smaller concentration to update to lower ranges the sensitivity of the Raman system. Each sample was tested at three different acquisition time: $5 \mathrm{~s}$ (corresponding on 20 accumulations on the ICCD), $12 \mathrm{~s}$ (50 accumulations) and $25 \mathrm{~s}$ (100 accumulations), respectively. A further consideration: the area of the laser beam $\left(0.785 \mathrm{~cm}^{2}\right)$ was slightly smaller than the area of the samples $\left(1.0 \mathrm{~cm}^{2}\right)$ : this factor was considered to calculate the exact amount probed. All the NATO-SET-237 samples were also measured by the laser microscope to verify the thickness of the explosives. 
Fig. 4 Sensitivity of RDX on NATO-SET-237 standard samples

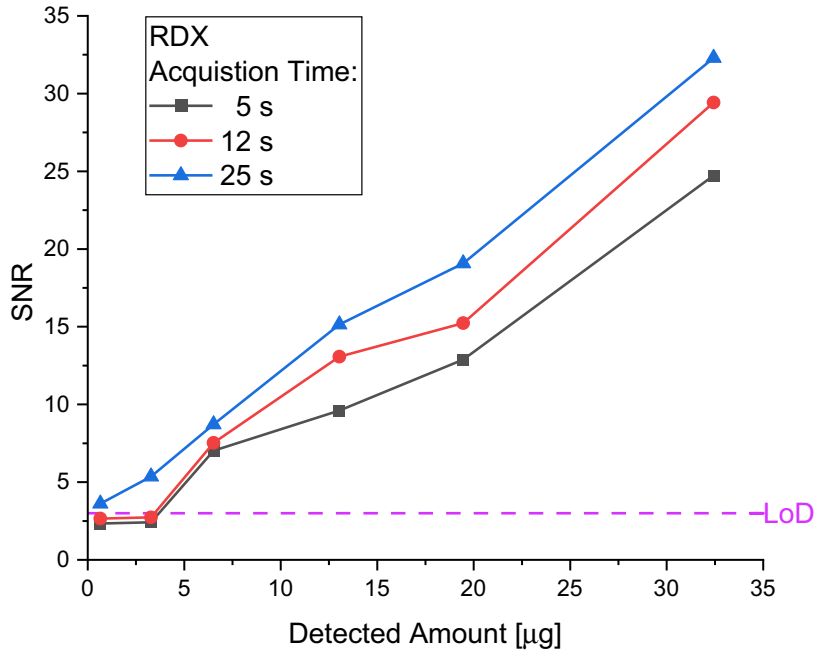

Table 4 Summary of minimum quantity of RDX detectable $\pm 4 \%$ on NATO-SET-237 standard samples

\begin{tabular}{llr}
\hline Acquisition Time $[\mathrm{s}]$ & Minimum Measured $[\mu \mathrm{g}]$ & Minimum Calculated $[\mu \mathrm{g}]$ \\
\hline 5 & 6.52 & 2.77 \\
12 & 6.52 & 2.11 \\
25 & 0.65 & 0.28 \\
\hline
\end{tabular}

Table 5 Summary of minimum quantity of TNT detectable $\pm 4 \%$ on NATO-SET-237 standard samples

\begin{tabular}{llr}
\hline Acquisition Time $[\mathrm{s}]$ & Minimum Measured $[\mu \mathrm{g}]$ & Minimum Calculated $[\mu \mathrm{g}]$ \\
\hline 5 & 2.18 & 1.86 \\
12 & 0.82 & 0.62 \\
25 & 0.82 & 0.24 \\
\hline
\end{tabular}

\subsubsection{RDX}

Figure 4 shows the sensitivity of RDX. It can be noticed that all the measurements were possible, even at the lowest concentration on the $1 \mu \mathrm{g} / \mathrm{cm}^{2}$ sample. The 25 -s acquisition time allows the detection of the smallest quantity of $0.65 \mu \mathrm{g}$ with a SNR of 3.6 which is still above the LoD. Looking at the three curves a linear behavior can be immediately noticed. Hence a liner interpolation was performed to theoretically estimate which was the lowest detectable amount. At SNR $=3$ the lowest possible detectable amount was $0.28 \mu \mathrm{g}$. Lowering the acquisition time decreases the signal strength, hence the SNR. So, the lowest tested concentration was below the LoD. The minimum measured amount was $6.52 \mu \mathrm{g}$ with 5 -s acquisition time. Again, the minimum quantity can be calculated using a linear interpolation, resulting to be $2.77 \mu \mathrm{g}$. Table 4 summarizes the sensitivity limits of RDX as function of acquisition time.

\subsubsection{TNT}

Figure 5 shows the sensitivity of TNT. As for RDX, it can be noticed that all the measurements were possible, even at the lowest concentration on the $1 \mu \mathrm{g} / \mathrm{cm}^{2}$ sample. The 25 -s acquisition time allows the detection of the smallest quantity of $0.82 \mu \mathrm{g}$ with a SNR of 4.9 which is above the LoD. Looking at the three curves, a logarithmic behavior can be immediately noticed. Hence a logarithmic interpolation was performed to theoretically estimate which was the lowest detectable amount. At SNR $=3$ the lowest possible detectable amount was $0.24 \mu \mathrm{g}$. The same procedure can be applied at the other acquisition times. Looking at the 5-s curve, the minimum measured quantity was $2.18 \mu \mathrm{g}$, but, using the theoretical calculation down to the LoD, the sensitivity drops to $1.86 \mu \mathrm{g}$.

Table 5 summarizes the sensitivity limits of RDX as function of acquisition time. 
Fig. 5 Sensitivity of TNT on NATO-SET-237 standard samples

Fig. 6 RDX NATO-SET-237

standard samples \#834 and \#837. Numbers in red represent the Raman peaks according to the literature
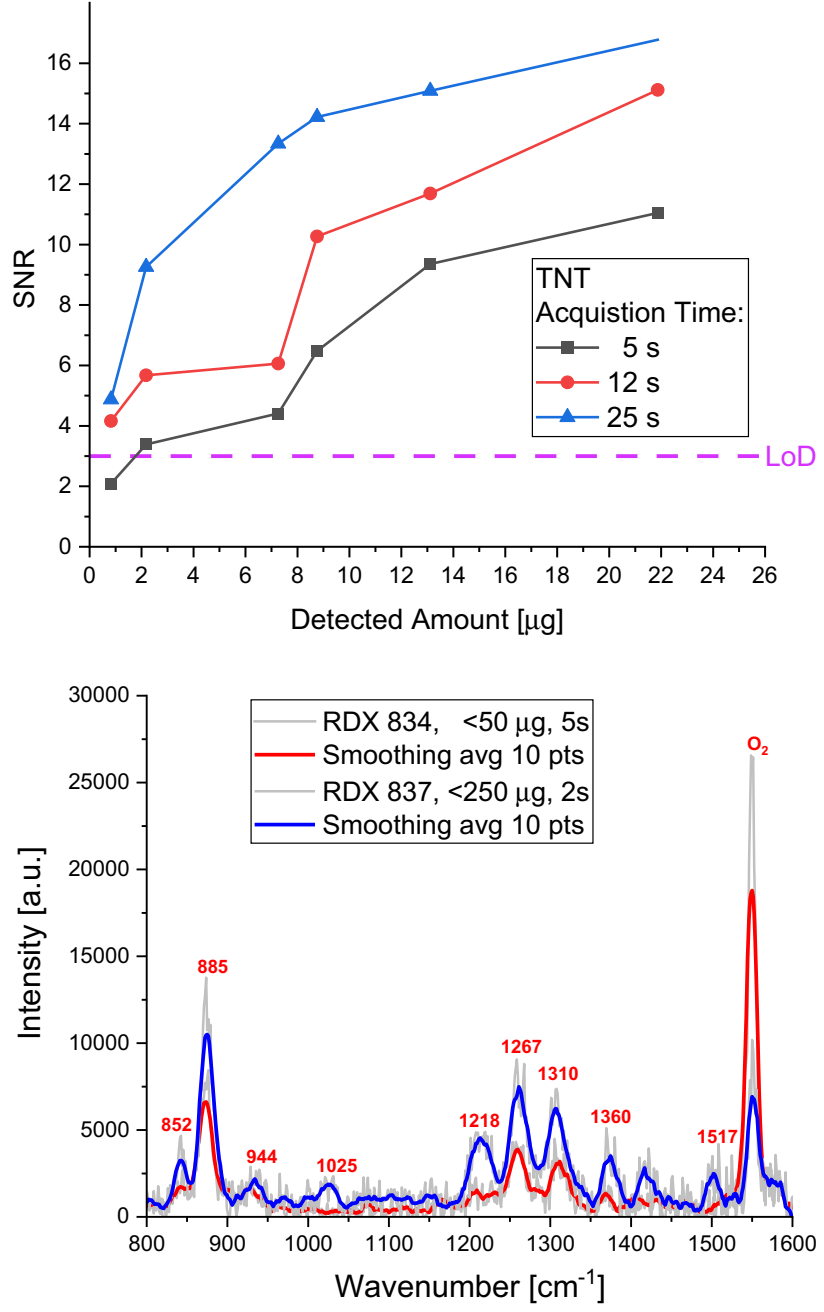

\subsection{Explosives on realistic background samples}

The experimental conditions used for the NATO-SET-237 standard samples were kept the same to probe the explosives on the realistic backgrounds. For each background 3 measurements were taken in different locations to probe different quantities. The exact amount of explosives detected was then determined in the post-processing, as described in details in data analysis section. The acquisition time was not constant but was changed to the minimum required to have a processable signal: it varied from a minimum of $1 \mathrm{~s}$ to a maximum of $25 \mathrm{~s}$. All the measurements were taken with the laboratory lights on.

\subsubsection{RDX}

Comparing to the NATO-SET-237 samples, the acquisition of Raman spectra on realistic backgrounds was more challenging. The distribution was completely non-uniform, and most of the background offered uneven surfaces and fluorescence interference. Therefore, the spectra acquired were not always clear, but they were instead very noisy. Hence, for a better visualization (not for the analysis), a smoothing function consisting on an average of 10 consecutive points was applied to each spectrum. Since plotting every single result might be space consuming, only a selected representative spectrum of each category (metals, construction, natural, transparent and plastics) will be shown. Final results, detected amount and sensitivity study however have been conducted for each individual tested background.

Figure 6 shows the spectrum of RDX on the NATO-SET-237 (ideal conditions) as reference in comparison with the real backgrounds that will follow. These samples, RDX \#834 and \#837, were old deteriorated samples with a high nominal concentration of 250 and $50 \mu \mathrm{g} / \mathrm{cm}^{2}$, respectively, that were used only to locate the Raman signature of RDX and not for further data analysis.

Figure 7 shows some selected spectra on realistic backgrounds. It can be immediately noticed that the baseline of the spectra is much noisier than the standard samples. For example, the brown paint provided some fluorescence that raised significantly the background noise. Contrary the brick provided almost no interference and the spectrum looks quite clear. The stone background 

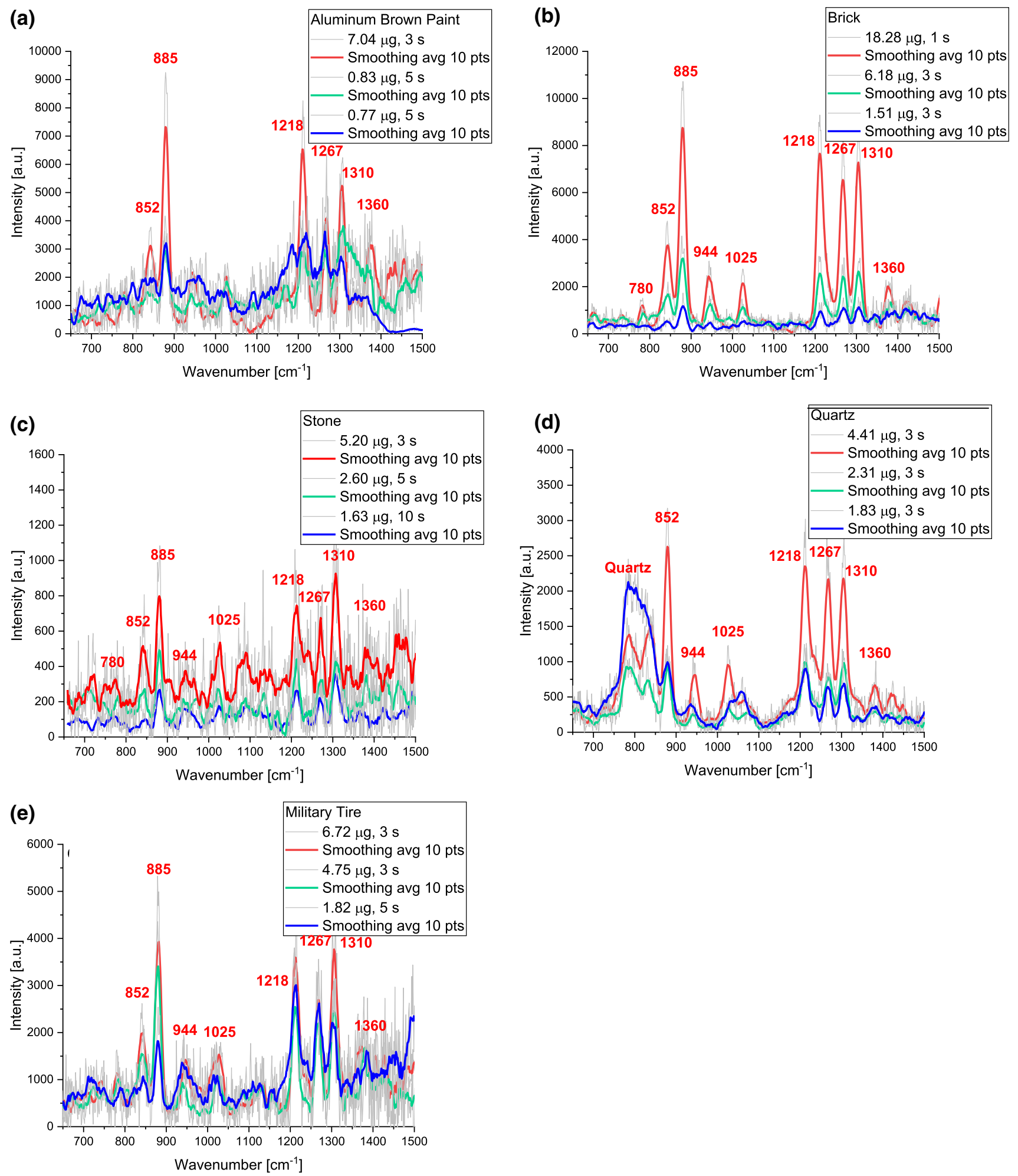

Fig. 7 RDX on selected realistic background samples: a brown aluminum paint; $\mathbf{b}$ brick; $\mathbf{c}$ stone; $\mathbf{d}$ quartz, $\mathbf{e}$ military tire. Numbers in red represent the RDX Raman peaks according to the literature

shows a very noisy baseline, probably due to some impurities deposited on the surface. Quartz, as expected, released its own Raman signal causing some signal overlapping in the $800 \mathrm{~cm}^{-1}$ region. The RDX spectrum on the tire is quite clear, and most of the peaks are distinguishable. It is also important to notice that the quantity of RDX detected is very small: most of the cases is below $10 \mu \mathrm{g}$. 
Also, the average acquisition time is within $5 \mathrm{~s}$. Another important observation is that the strongest peak at $885 \mathrm{~cm}^{-1}$ is always noticeable, and this peak is relevant because it was used for the SNR calculation.

Figure 8 shows the sensitivity of RDX on realistic backgrounds by each material category. It is important to remind that the SNR is calculated with respect to the $885 \mathrm{~cm}^{-1}$ peak. It can be noticed that all the measurements performed are above the LoD (LoD threshold is marked as pink horizontal dashed line in each picture below). In addition, all the quantities detected are below $20 \mu \mathrm{g}$. Some consideration must be drawn based on the results; all the metals provided a good SNR despite the colored paint that creates some fluorescence interference. The minimum detected amount was $0.76 \mu \mathrm{g}$ on brown painted aluminum. Construction materials are grouped with material with different properties, while brick offers almost no interferences (as shown on the spectrum in Fig. 8b); the other materials provided some challenges. Asphalt has a very rough surface, in addition to petroleum matrix which causes some fluorescence. Concrete provided a stronger signal comparing asphalt, mostly due to its smoother surface. Cardboard was limited by a strong fluorescence that saturates the camera immediately: only $1 \mathrm{~s}$ of acquisition time was allowed to avoid saturation. The same argumentation is valid for linoleum (mostly due to the colored pattern) and rubber.

As already done for the standard samples, mathematical interpolation can be used to fit each individual line and identify the minimum detectable amount (above LoD) for each background. Of course, since the materials are so different from each other, it is not possible to use a unique function, so some lines would use a linear, logarithmic or exponential fit. Whenever the interpolation line provided a negative minimum value or not enough measurement points were successful to perform the analysis, only the smallest amount measured was reported. The summary of the results is found in Table 6.

It can be noticed that only cardboard has a SNR slightly below the LoD when probed for $5 \mathrm{~s}$; therefore, at least $6 \mathrm{~s}$ is advised. The minimum measured quantities are all below $10 \mu \mathrm{g}$ (smallest $760 \mathrm{ng}$ detected on brown paint aluminum). These results placed already the presented system developed within the group of the best sensitivity for a remote detection system worldwide. The mathematical interpolation proves that those limits can be further pushed down in the range below $1.0 \mu \mathrm{g}$, where no publications were found except for close by microscopic analysis (here the downside is direct contact between the operator and the probed material) or with methodology that require a sample preparation (such a mass spectrometer analysis).

\subsubsection{TNT}

The description of the TNT follows the same methodology as the RDX. TNT provided additional challenges because of the fast photodissociation [15]. Therefore, all the tests with TNT were performed with the minimal exposure time of the ICCD. In other words, increasing the acquisition time does not increase the SNR because TNT would be deteriorated in few seconds. This issue happened with all the laser sources tested and TNT photo-dissociation also occurs with sun exposure too [15]. Figure 9 shows the spectrum of TNT on the NATO-SET-237 (ideal conditions) as reference in comparison with the real background that will follow. This sample, TNT \#850, was an old deteriorated sample with nominal high concentration of $1000 \mu \mathrm{g} / \mathrm{cm}^{2}$ that was used only to identify the Raman signature of TNT and not for further data analysis.

Figure 10 shows some selected spectra on realistic backgrounds, each representative of one category. It can be immediately noticed that the all the spectra are much noisier comparing RDX. This is mainly due to the faster acquisition time which it is necessary to avoid photo-degradation. A strong fluorescence signal is present for the wood material tested (beech bark, Fig. 10c), so, for these samples, the portion available for identification of TNT is before $1500 \mathrm{~cm}^{-1}$. A clear example of photo-degradation is shown in Fig. 10e: acquiring the first data (green curve) the TNT features are distinguishable while repeating the measurement was not successful (red curve) because all sample was degraded. This led to the conclusion that when testing TNT only one test can be performed before degradation occurs. Even when Plexiglas (Fig. 10d) shows its own Raman signal, it was possible to discriminate which peak belongs to Plexiglas and which one to TNT. In this case, one peak is available for detecting TNT: $1483 \mathrm{~cm}^{-1}$. The remaining tested backgrounds were painted aluminum and asphalt and provided a good SNR.

Figure 11 shows the sensitivity of TNT on realistic backgrounds by each material category. It is important to remind that the SNR was calculated with respect to the $1483 \mathrm{~cm}^{-1}$ peak. Due to the limited acquisition time not all the backgrounds provided successful measurements. In few cases it was possible to increase the acquisition time till the complete photo-dissociation of TNT, as described for the synthetic leather. In other cases, increasing the time led to a stronger interference fluorescence which masked completely the Raman signal. This is the case for rubber, pine bark and wood generally, which are not presented for this reason. Stone did not offer adherence for the TNT over its surface and for this reason was not detected. SNR for cardboard was 2.5, which is below LoD so even if the amount detected was $2.13 \mu \mathrm{g}$, the data were not considered. Despite some limitations for wood, natural rubber (vehicle tires work instead) and cardboard, it can be noticed that most of the successful measurements are above the LoD. In addition, all the quantities detected are below $30 \mu \mathrm{g}$. Some consideration must be drawn based on the results; all the metals provided a good SNR despite the colored paint that creates some fluorescence interference. Minimum detected amount was $0.67 \mu \mathrm{g}$ on black-painted aluminum.

As already done for RDX, mathematical interpolation can be used to fit each individual line and identify the minimum detectable amount (above LoD) for each background. Again, since the materials are so different from each other, is not possible to use a unique function, so some line would use a linear, logarithmic or exponential fit. The summary of the results is found in Table 7. It can be noticed that despite cardboard, where the SNR is slightly below the LoD, the minimum measured quantity is in the range of $10 \mu \mathrm{g}$ (smallest $670 \mathrm{ng}$ detected on black-painted aluminum). This result placed already the system developed within the group of the best 

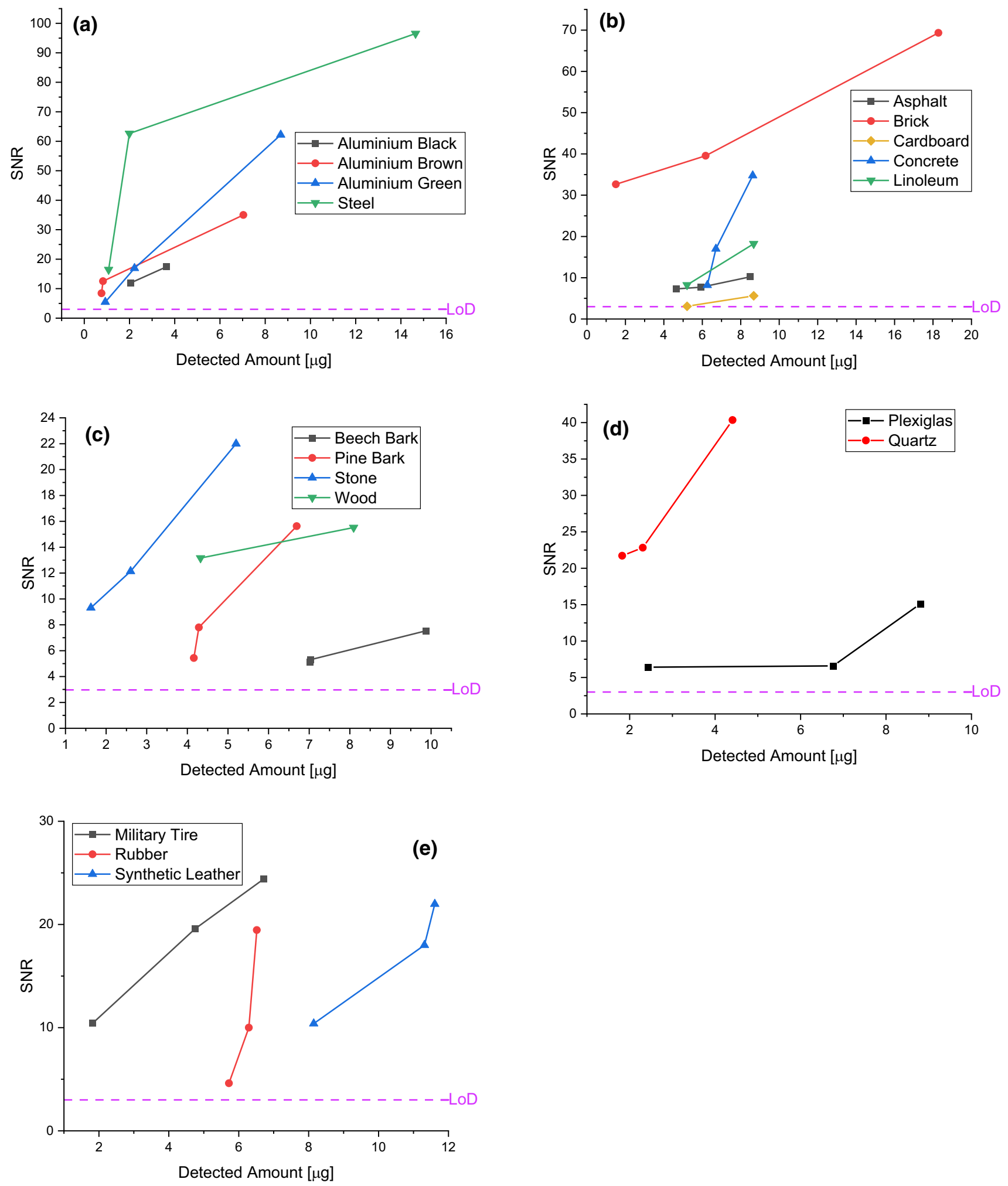

Fig. 8 Sensitivity of RDX on realistic backgrounds: a metals; $\mathbf{b}$ construction; $\mathbf{c}$ natural; $\mathbf{d}$ transparent; e plastics

sensitivity for a remote detection system worldwide. The mathematical interpolation proves that those limits can be further pushed down in the range below $1.0 \mu \mathrm{g}$, where no publications were found except for close by microscopic analysis (with the downside of being a direct contact method) or with methodology that requires a sample preparation (such a mass spectrometer analysis). 
Table 6 Summary of minimal quantity of RDX detectable $\pm 4 \%$ on realistic backgrounds

\begin{tabular}{llll}
\hline & Background & Minimum Measured $[\mu \mathrm{g}]$ & Minimum Calculated $[\mu \mathrm{g}]$ \\
\hline \multirow{2}{*}{ Metals } & Aluminum Black & 2.04 & 0.11 \\
& Aluminum Brown & 0.76 & 0.41 \\
& Aluminum Green & 0.93 & 0.64 \\
& Steel & 1.08 & - \\
Construction & Asphalt & 4.64 & - \\
& Brick & 1.51 & - \\
& Cardboard & 5.86 & 5.99 \\
& Concrete & 6.26 & 3.15 \\
Natural & Linoleum & 5.20 & 4.55 \\
& Beech Bark & 7.02 & 3.75 \\
& Pine Bark & 4.16 & 0.29 \\
Transparent & Stone & 9.31 & - \\
& Wood & 4.33 & - \\
Plastics & Plexiglas & 2.43 & - \\
& Quartz & 1.82 & 0.31 \\
& Military Tire & 1.82 & 5.32 \\
& Rubber & 5.72 & 4.63 \\
\hline
\end{tabular}

Fig. 9 TNT NATO-SET-237

standard sample \#850. Numbers in red represent the Raman peaks according to the literature

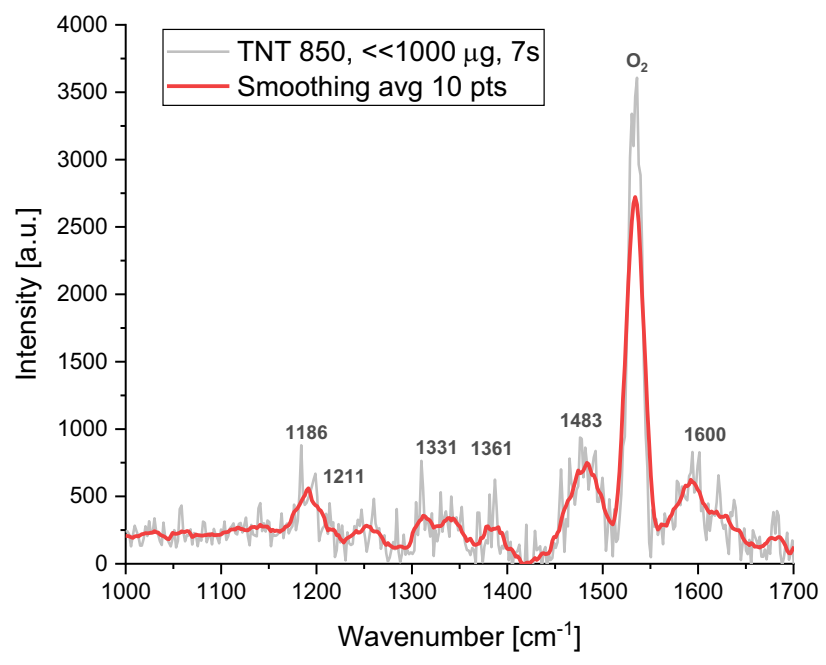

\subsection{Warfare agent simulants on realistic background samples}

The experimental conditions used for the explosives were kept the same to probe the warfare agent simulants on the realistic backgrounds. For each background 3 measurements were taken in different locations to probe different quantities. The exact amount of simulants detected was then determined in the post-processing, as described in details in the data analysis section. Note that the final quantities of the warfare agent simulants are bigger comparing explosives, since no literature was found to estimate the penetration depth. Therefore, the full thickness of each compound measured by the laser microscope was considered to evaluate the scattered volume. These values are known to be overestimated: it is not expected that the penetration depth of the warfare agent simulants exceeds the penetration depth of the human skin $(\sim 250 \mu \mathrm{m}$ in the deep UV range). However, it was decided to use the exact thickness in absence of a clear scientific publication or a proven methodology to measure the exact value. The acquisition time was not constant but was changed to the minimum required to have a processable signal: it varied from a minimum of $1 \mathrm{~s}$ to a maximum of $14 \mathrm{~s}$. All the measurements were taken with the laboratory lights on. 

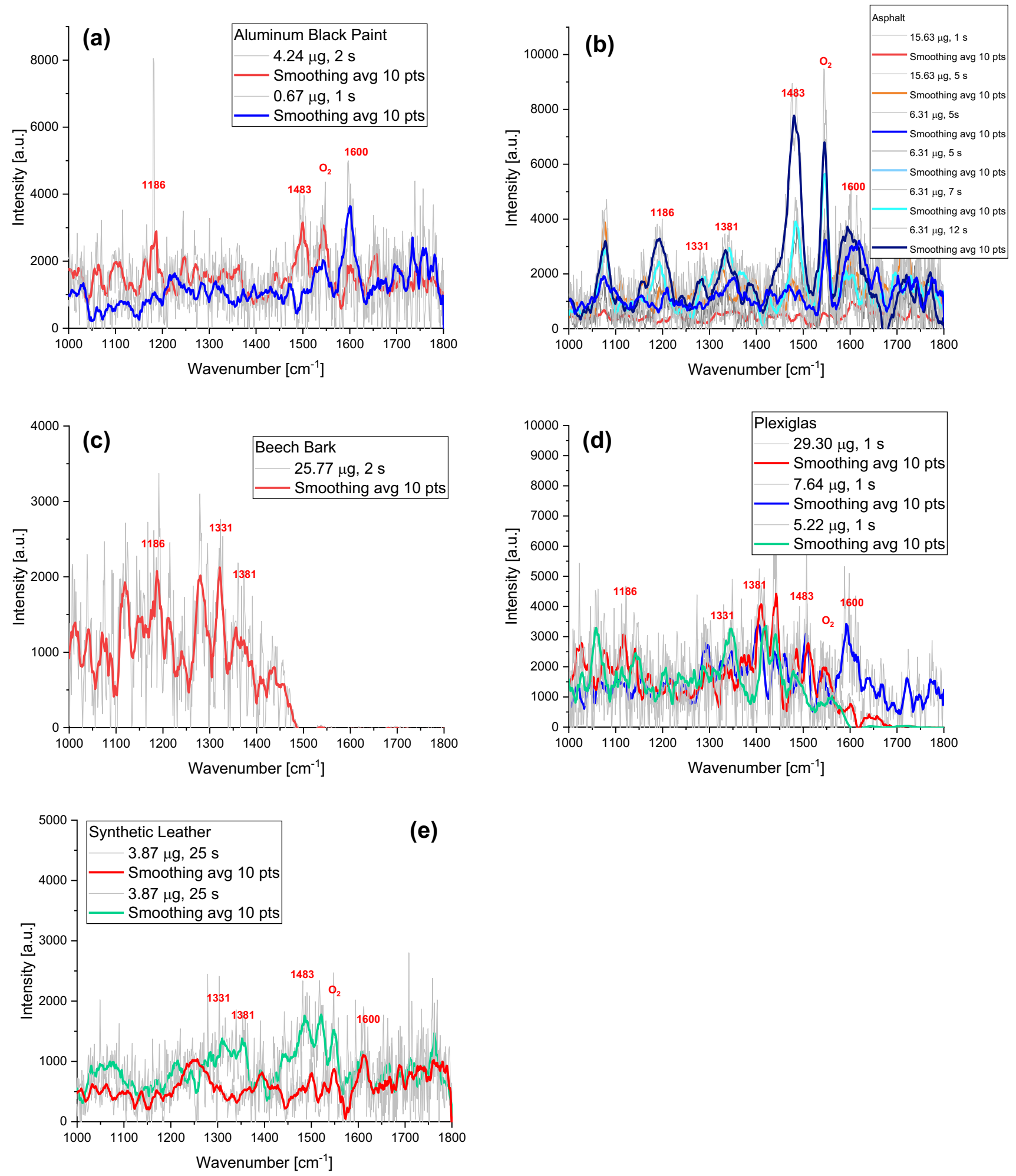

Fig. 10 TNT on selected realistic background samples: a black aluminum paint; $\mathbf{b}$ asphalt; $\mathbf{c}$ beech bark; d Plexiglas, e synthetic leather. Numbers in red represent the TNT Raman peaks according to the literature

\subsubsection{GLP}

A similar discussion to the explosive section can be applied to this chapter. Again, the distribution of the material was completely non-uniform, and most of the background offered uneven surfaces and fluorescence interference: this was the main challenge of the 

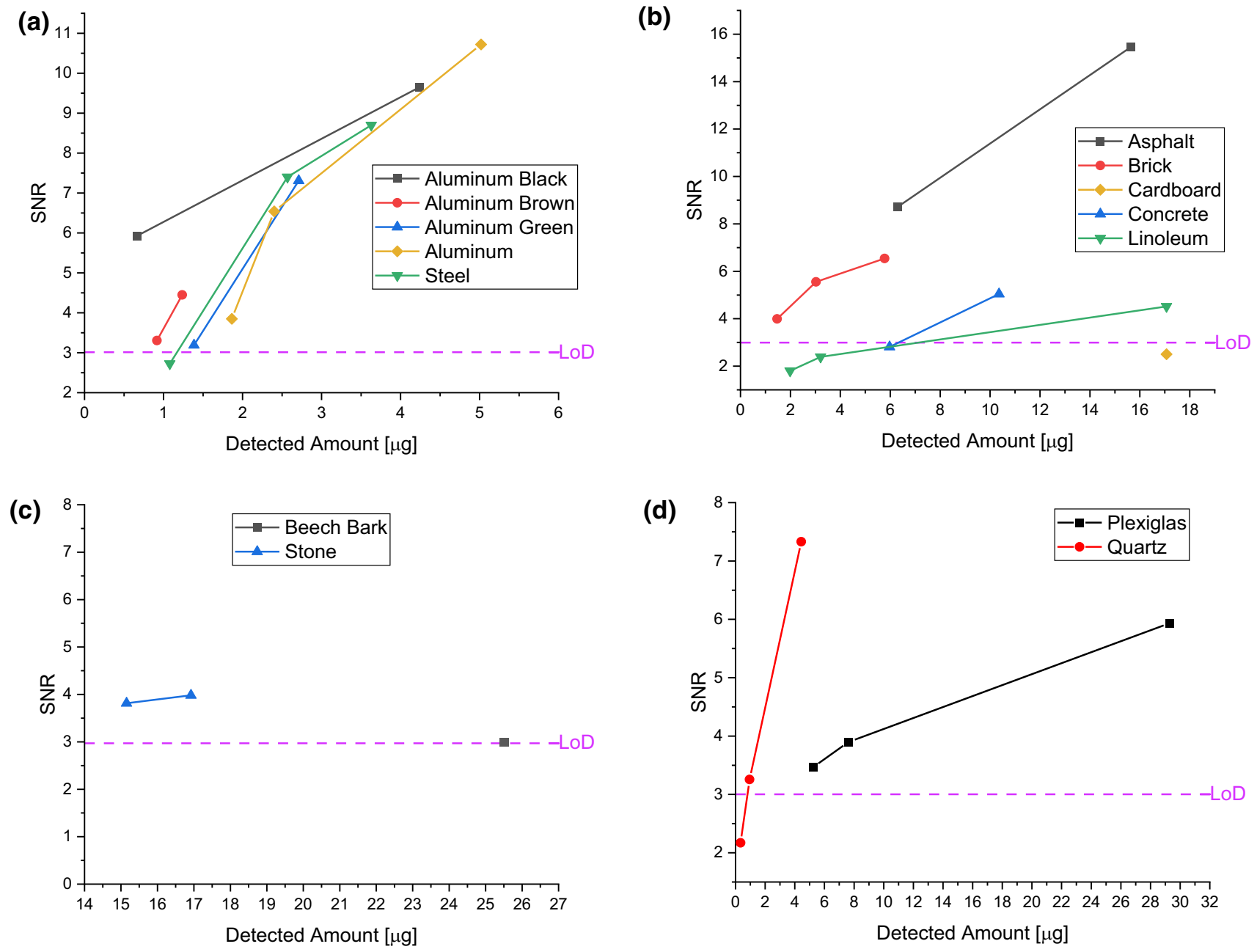

(e)

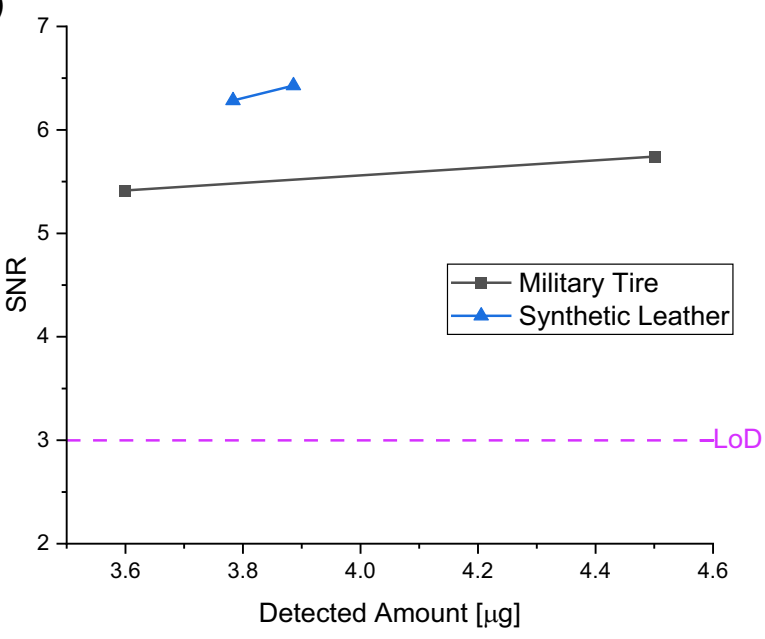

Fig. 11 Sensitivity of TNT on realistic backgrounds: a metals; $\mathbf{b}$ construction; $\mathbf{c}$ natural; $\mathbf{d}$ transparent; $\mathbf{e}$ plastics

experiments. Therefore, the spectra acquired showed some background interference. Hence, for a better visualization, a smoothing function consisting on an average of 10 consecutive points was applied to each spectrum. Since plotting every single result might be space consuming, only a selected representative spectrum of each category (metals, construction, natural, transparent and plastics) will be shown. Final results, detected amount and sensitivity study however were performed for each individual tested background.

Figure 12 shows the spectrum of bulk GLP (ideal conditions) inside a Suprasil cuvette (an empty quartz cell reference spectrum was also recorded, and its peaks were labeled). The recorded bulk reference spectrum is shown to identify all the significant peaks 
Table 7 Summary of minimal quantity of TNT detectable $\pm 4 \%$ on realistic backgrounds

\begin{tabular}{llll}
\hline & Background & Minimum Measured $[\mu \mathrm{g}]$ & Minimum Calculated $[\mu \mathrm{g}]$ \\
\hline \multirow{2}{*}{ Metals } & Aluminum Black & 0.67 & 0.12 \\
& Aluminum Brown & 0.92 & 0.84 \\
& Aluminum Green & 1.39 & 1.31 \\
& Aluminum & 1.86 & 1.73 \\
& Steel & 2.57 & 1.17 \\
Construction & Asphalt & 6.31 & 0.42 \\
& Brick & 1.47 & 0.95 \\
& Cardboard & - & - \\
& Concrete & 10.36 & 6.35 \\
Natural & Linoleum & 17.07 & 7.34 \\
& Beech Bark & 25.77 & - \\
Transparent & Stone & 15.15 & 8.53 \\
& Plexiglas & 5.22 & 3.98 \\
Plastics & Quartz & 0.94 & 0.80 \\
& Military Tire & 3.60 & - \\
\hline
\end{tabular}

Fig. 12 Bulk GLP spectrum inside a Suprasil cuvette. Numbers in red represent the Raman peaks according to the literature. Black arrows show the common peaks with Sarin

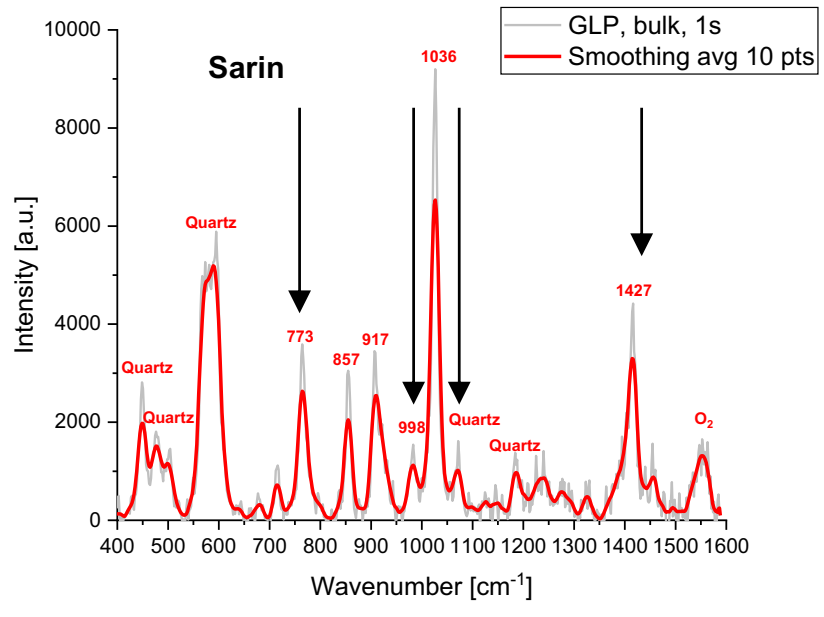

in comparison with the real backgrounds that will follow. Since GLP was used as a simulant of Sarin, all the common peaks with the nerve agent are marked with a black arrow.

Figure 13 shows some selected spectra of GLP on realistic backgrounds, each representative of one category. It can be observed that the GLP spectra are always well defined regardless of the background. This led to a very good SNR. However, the focus must be on the few peaks representing the Sarin nerve agent and marked in red with numbers. Comparing to Fig. 12, it can be noticed that all the peaks representing Sarin are always well captures by system; therefore, the required selectivity is always met. Particularly, it is important to focus on the peak at $773 \mathrm{~cm}^{-1}$ which is used to calculate the SNR of Fig. 13. This peak is always visible regardless of the background noise proving good confidence about the identification.

Figure 14 shows the sensitivity of Sarin (extrapolated from GLP) on realistic backgrounds by each material category. It can be immediately noticed that all the measurements are above the LoD with a very good SNR. A first explanation could due to the higher concentration of GLP on the samples. This statement is partially true: from one side the total material deposited and detected by the Raman system is higher comparing to explosives. However, it must be considered that the penetration depth is unknown for GLP, and the measured thickness overestimated the scattering volume that generates the signal. This effect is particularly evident on very groovy or rough materials such asphalt, concrete, brick or barks, where a lot of GLP can accumulate in a single location. Despite this overestimation of the quantity, it is important to underline that the signal was obtained within $2 \mathrm{~s}$ for most of the measurements. This quick response makes the system able to provide a live monitoring for this kind of chemical substance.

As already done for the explosive materials, mathematical interpolation can be used to fit each individual line and identify the minimum detectable amount (above LoD) for each background. Again, since the materials are so different from each other, it is 

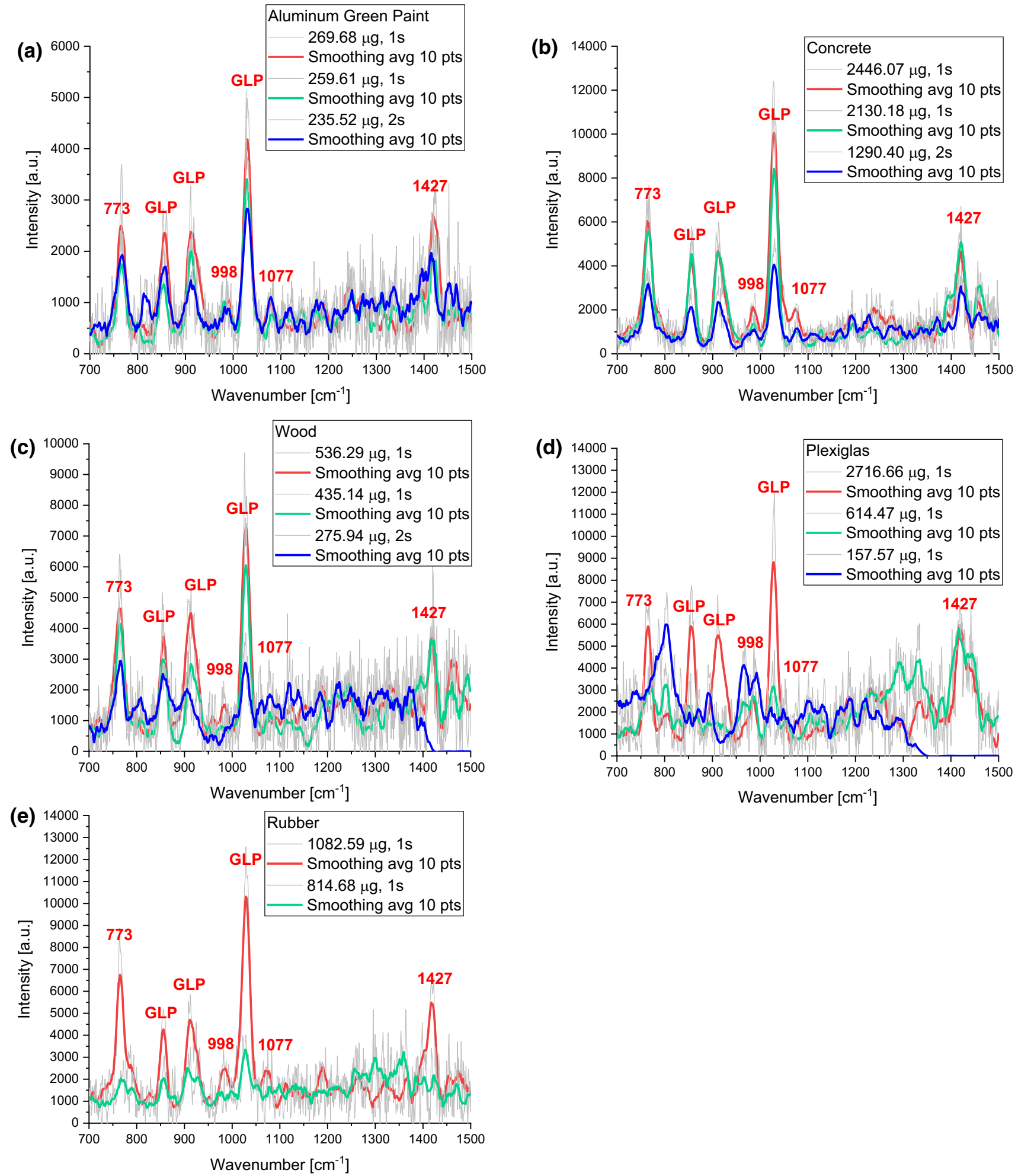

Fig. 13 GLP on selected realistic background samples: a green aluminum paint; $\mathbf{b}$ concrete; $\mathbf{c}$ wood; $\mathbf{d}$ Plexiglas, $\mathbf{e}$ rubber. Numbers in red represent the Sarin Raman peaks according to the literature

not possible to use a unique function, so some line would use a linear, logarithmic or exponential fit. The summary of the results is found in Table 8. For Sarin, the mathematical interpolation plays a bigger role. Since most of the tests performed provided a very high SNR, it is important to find out which is the realistic limit of the technique. Most of the calculated limit, except few cases where only one single measurement was possible (hence, not enough information for a mathematical interpolation), and rubber, all the 

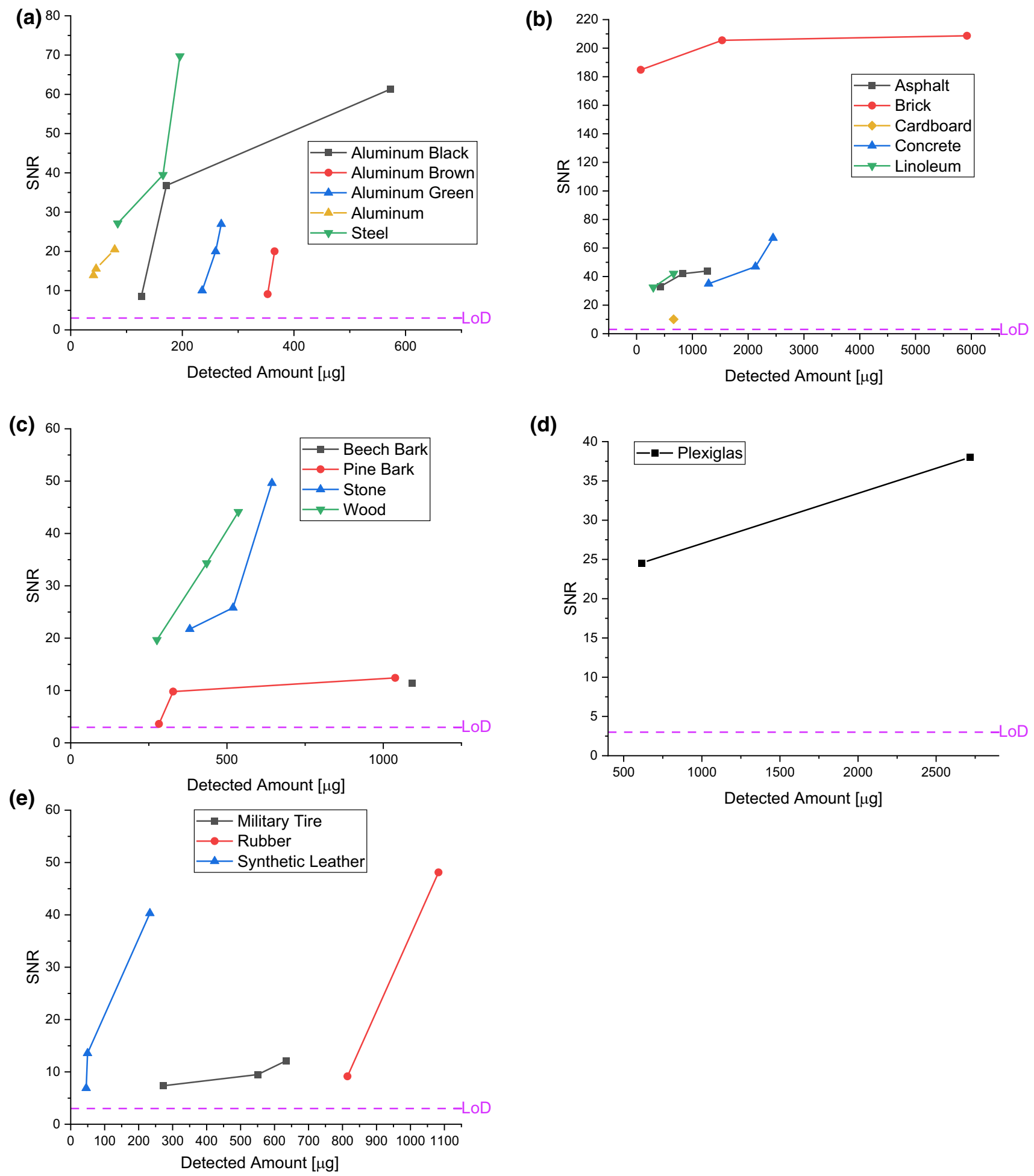

Fig. 14 Sensitivity of Sarin (extrapolated from GLP) on realistic backgrounds: a metals; b construction; c natural; d transparent; $\mathbf{e}$ plastics

measurements are below the $500 \mu \mathrm{g}$ (and 20 min exposure) which is the toxicology limit [26]. This result provides the confidence that this methodology is able to detect Sarin and to raise an alarm before the effects of the nerve agent may become dangerous (or deadly). 
Table 8 Summary of minimal quantity of Sarin (extrapolated from GLP) detectable $\pm 6 \%$ on realistic backgrounds

\begin{tabular}{llcc}
\hline & Background & Minimum Measured $[\mu \mathrm{g}]$ & Minimum Calculated $[\mu \mathrm{g}]$ \\
\hline \multirow{2}{*}{ Metals } & Aluminum Black & 126.78 & 116.19 \\
& Aluminum Brown & 352.92 & 344.57 \\
& Aluminum Green & 235.52 & 206.99 \\
& Aluminum & 40.87 & 10.37 \\
& Steel & 27.14 & - \\
Construction & Asphalt & 420.43 & 44.23 \\
& Brick & 73.98 & - \\
& Cardboard & 554.45 & - \\
& Concrete & 1290.40 & 26.67 \\
Natural & Linoleum & 32.46 & - \\
& Beech Bark & 1092.82 & - \\
& Pine Bark & 282.56 & 273.60 \\
Transparent & Stone & 380.91 & 13.67 \\
Plastics & Wood & 275.94 & 102.03 \\
& Plexiglas & 614.48 & - \\
& Military Tire & 272.31 & 18.35
\end{tabular}

Fig. 15 Bulk TPP spectrum inside a Suprasil cuvette. Numbers in red represent the Raman peaks according to the literature. Black arrows show the common peaks with VX

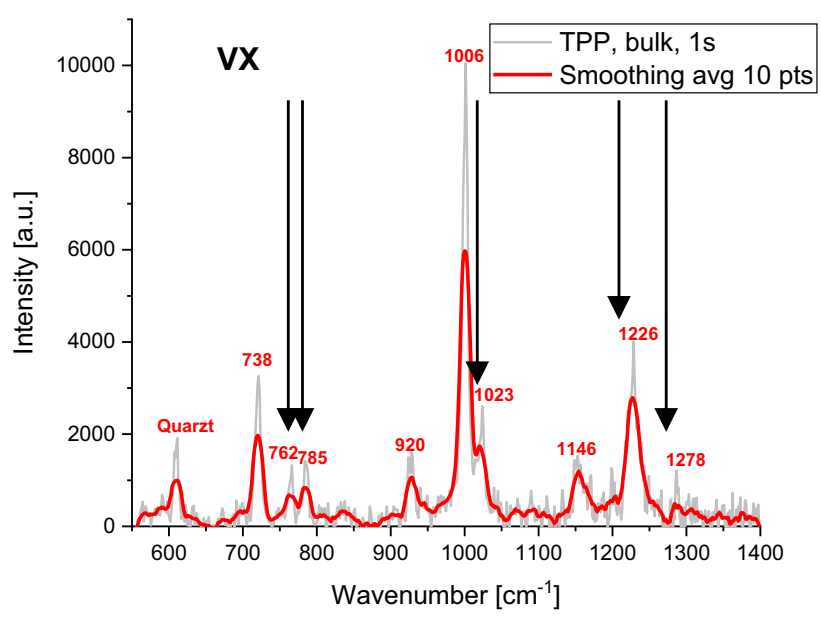

\subsubsection{TPP}

All the results hereby presented were obtained with the same operating conditions and methodology of the previous chapters. Figure 15 shows the spectrum of bulk TPP (ideal conditions) inside a Suprasil cuvette (possible overlapping quartz spectrum was also recorded its peak labeled). It is shown as a reference spectrum to identify all the significant peaks in comparison with the real backgrounds that follow. Since TPP was used as a simulant of VX, all the common peaks with the nerve agent are marked with a black arrow.

Figure 16 shows some selected spectra of GLP on realistic backgrounds, each representative of one category. It can be observed that the TPP spectra are always well defined regardless of the background. This led to a very good SNR. However, the focus must be on the few peaks representing the VX nerve agent and marked in red. Similar discussions and conclusions to GLP can be done with TPP: all the peaks representing VX are always well captures by system; therefore, the required selectivity is always met. Particularly, it is important to focus on the peak at $1226 \mathrm{~cm}^{-1}$ which was used to calculate the SNR. This peak is always visible regardless of the background noise (particularly noisy was Plexiglas, due to its own Raman signal, and military tire or in general plastics, where strong fluorescence was detected) proving good confidence about the identification.

Figure 17 shows the sensitivity of VX (extrapolated from TPP) on realistic backgrounds by each material category. It is important to remind that the SNR is calculated with respect to the $1226 \mathrm{~cm}^{-1}$ peak. 

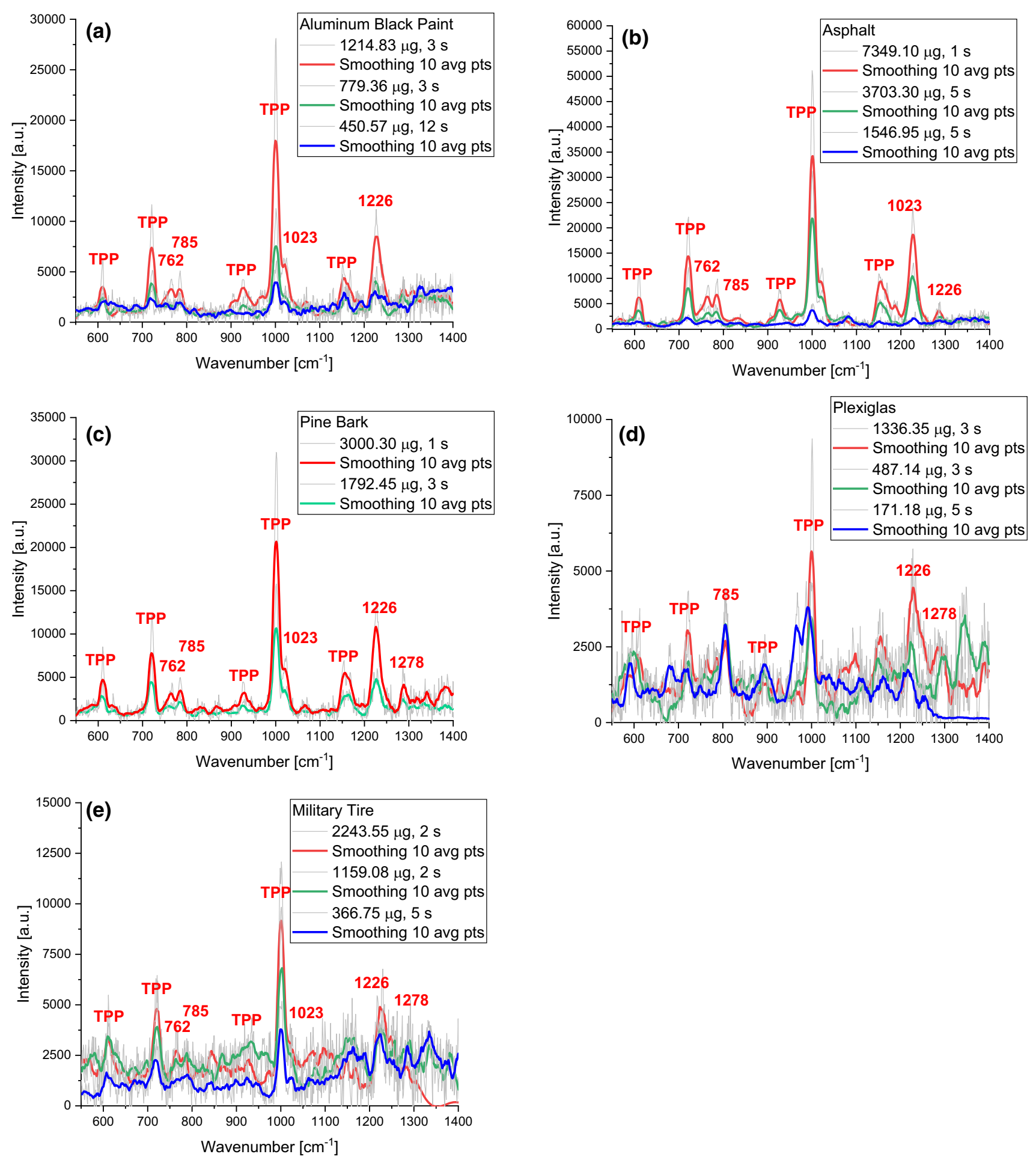

Fig. 16 TPP on selected realistic background samples: a black aluminum paint; $\mathbf{b}$ asphalt; $\mathbf{c}$ pine bark; $\mathbf{d}$ Plexiglas, e military tire. Numbers in red represent the VX Raman peaks according to the literature

It can be immediately noticed that, also for VX, all the measurements are above the LoD with a very good SNR within 5-s acquisition time. As mentioned before in the Sarin discussion, it must be considered that the penetration depth is unknown for TPP, and the measured thickness overestimated the scattering volume that generates the signal. Despite this overestimation of the quantity, it is important to underline that the signal was obtained within $5 \mathrm{~s}$. The summary of the detectable quantities is found in Table 9.

As already done in the previous chapters, mathematical interpolation can be used to fit each individual line and identify the minimum detectable amount (above LoD) for each background. For VX too, the mathematical interpolation plays a bigger role. 

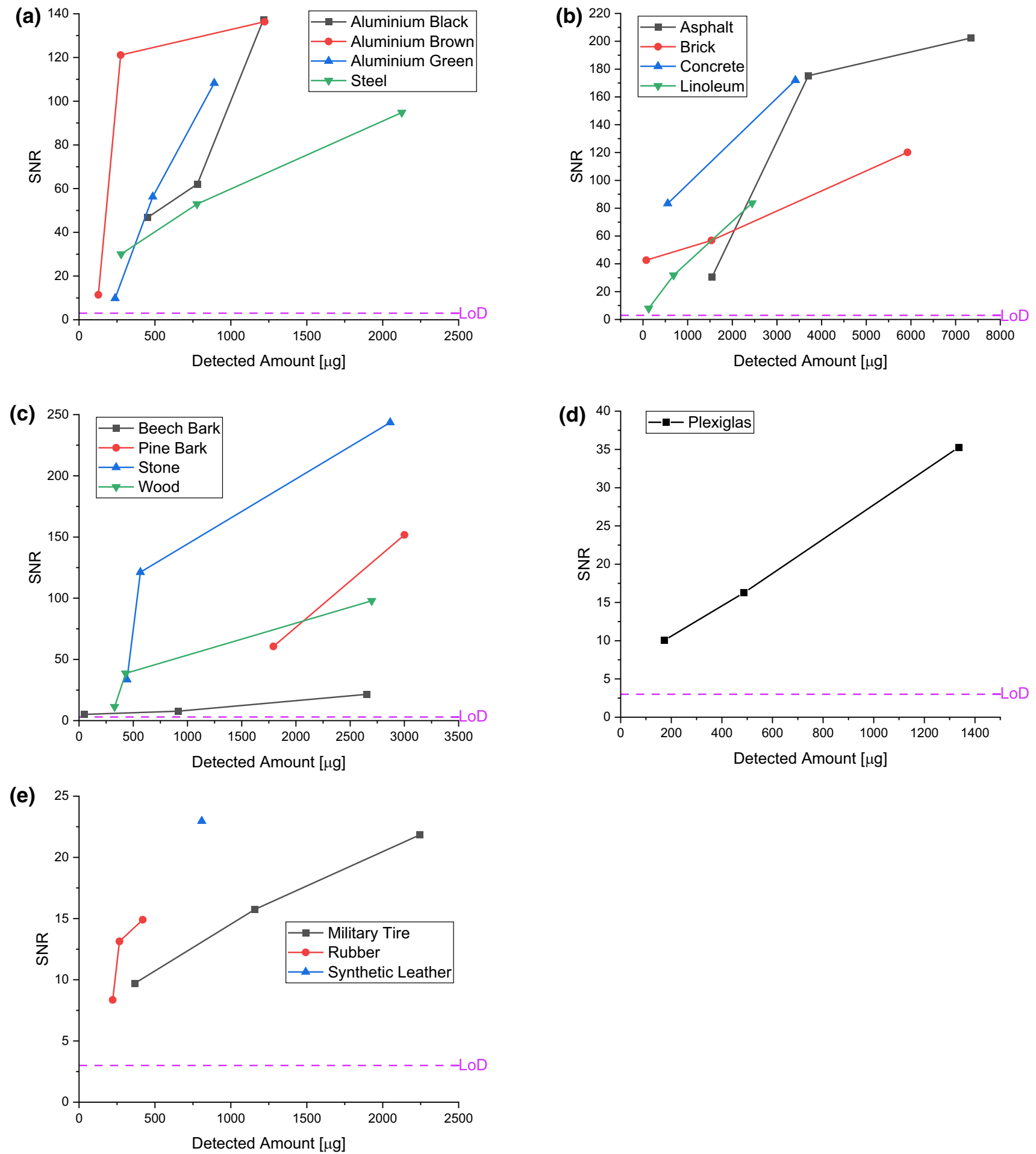

Fig. 17 Sensitivity of VX (extrapolated from TPP) on realistic backgrounds: a metals; $\mathbf{b}$ construction; $\mathbf{c}$ natural; $\mathbf{d}$ transparent; e plastics

Since most of the tests performed provided a very high SNR, it is important to find out which are the capabilities and limitations of the Raman technique. Most of the calculated limit, except few cases where only one single measurement was possible (hence, not enough information for a mathematical interpolation), asphalt and pine bark (where a lot of material can accumulate inside the uneven surface), all the measurements are below the $500 \mu \mathrm{g}$ (and $20 \mathrm{~min}$ exposure) [26] which is the toxicology limit. This result provides the confidence that this methodology is able to detect VX and to raise an alarm before the effects of the nerve agent may become dangerous (or deadly). 
Table 9 Summary of minimal quantity of VX (extrapolated from TPP) detectable $\pm 6 \%$ on realistic backgrounds

\begin{tabular}{llcc}
\hline & Background & Minimum Measured $[\mu \mathrm{g}]$ & Minimum Calculated $[\mu \mathrm{g}]$ \\
\hline \multirow{2}{*}{ Metals } & Aluminum Black & 450.59 & - \\
& Aluminum Brown & 127.27 & 106.02 \\
& Aluminum Green & 237.61 & 204.06 \\
& Steel & 276.82 & 14.82 \\
Construction & Asphalt & 1546.95 & 1220.23 \\
& Brick & 73.98 & - \\
& Concrete & 553.94 & - \\
Natural & Linoleum & 128.81 & 11.63 \\
& Beech Bark & 50.01 & 11.96 \\
& Pine Bark & 1792.45 & 1145.12 \\
Transparent & Stone & 442.48 & 369.47 \\
Plastics & Wood & 328.17 & 289.67 \\
& Plexiglas & 171.18 & - \\
& Military Tire & 366.75 & 18.23 \\
& Rubber & 221.88 & 175.56 \\
\hline
\end{tabular}

\subsection{Incident laser beam angle dependency}

A series of tests were performed to investigate how the angle of the incident laser beam can affect the detection of hazardous substances. This test is interesting for understanding how the Raman system responds in a realistic scenario in case the laser system will be set at a different angle with respect to the targeting sample. For this purpose, a sample holder capable to rotate around its own axis was built and employed. In this way, several angles can be simulated and therefore evaluated which were the limit of the detection on the system when it was off from the optimal configuration. Two different chemicals were tested: RDX as representative of the explosives and GLP (simulant of Sarin) as representative of warfare agent simulants. An additional variable was added to the test: two different backgrounds were considered. A stainless steel plate was used as a clean non-interference surface as used as an ideal reference background; the green paint aluminum plate was chosen since it provided fluorescence interference. For the latter background it is important to check how much the angle of the incident laser beam affects the strength of the fluorescence, hence creating a disturbance to detect the Raman signal. Uneven and rough surfaces were not considered for this test because all of the uncertainties related to the angle evaluation. All the tests were performed using self-made samples: small amount $(<1 \mathrm{mg})$ of RDX and GLP were compacted on both surfaces to produce a round shape of $0.5 \mathrm{~cm}$ diameter. The amount of compounds were chosen such that the Raman signal was strong enough to be detected at any angle change and decoupled from the concentration. In this case, the SNR was expected to be higher than the previous measurements, since the interest was focused only on the angle change dependency. The 355-nm excitation laser beam was kept constant for the entire campaign: the round laser beam had a size of $1.0 \mathrm{~cm}$ diameter with a constant energy of $8.0 \mathrm{~mJ} /$ pulse at $20 \mathrm{~Hz}$ pulse rate. The angles were varied from $-60^{\circ}$ to $+60^{\circ}$, and measurements were taken every $10^{\circ}$. Positive angles are in clock direction, while negative angles in counter clock direction. All the angles were measured with respect to the optimal configuration $\left(0^{\circ}\right)$ at which all the previous described tests were performed.

Figure 18a shows the results for RDX. It can be immediately noticed that the peak of the signal is located at $0^{\circ}$ for the green-painted aluminum, while for the stainless steel plate is shifted $10^{\circ}$ backward. This result was not a surprise: in an ideal configuration the maximum of the Raman signal is expected normal to the incident light. However, once interferences are expected, a compromise must be found. In this case tilting a little bit the sample or changing slightly the angle of the excitation laser beam may result in an increase of the signal and/or removal/decreasing of the interferences. Therefore, there is not a unique angle that can be applied for all the surfaces and all the target materials but a range for which the measurements lay into the optimal region. In this study this optimal range is located between $-10^{\circ}$ and $+10^{\circ}$. Looking again at Fig. 18a, it can be inferred easily that the stainless steel provided a stronger SNR, hence a stronger signal. This result was expected since the stainless steel does not generate any native fluorescence at $355 \mathrm{~nm}$, while the green paint does. This added extra interference affected the signal strength, therefore the lower SNR curve on the green line. It is also important to notice also that in both cases even when the sample is rotated outside the optimal range, the SNR is still high enough to be always above the LoD. In fact, even at the extremes of the measurements $\left( \pm 60^{\circ}\right)$ the SNR is always above the LoD. So, it can be inferred that the Raman technique is angle dependent but not as strong as other laser-based techniques as for example mid-infrared spectroscopy [1].

Figure 18b shows the same tests performed with Sarin (extrapolated by GLP). Also, in this case the optimal range of measurements lies between $-10^{\circ}$ and $+10^{\circ}$. The difference in strength of the signal is also noticeable between fluorescing and non-fluorescing 


\section{(a)}

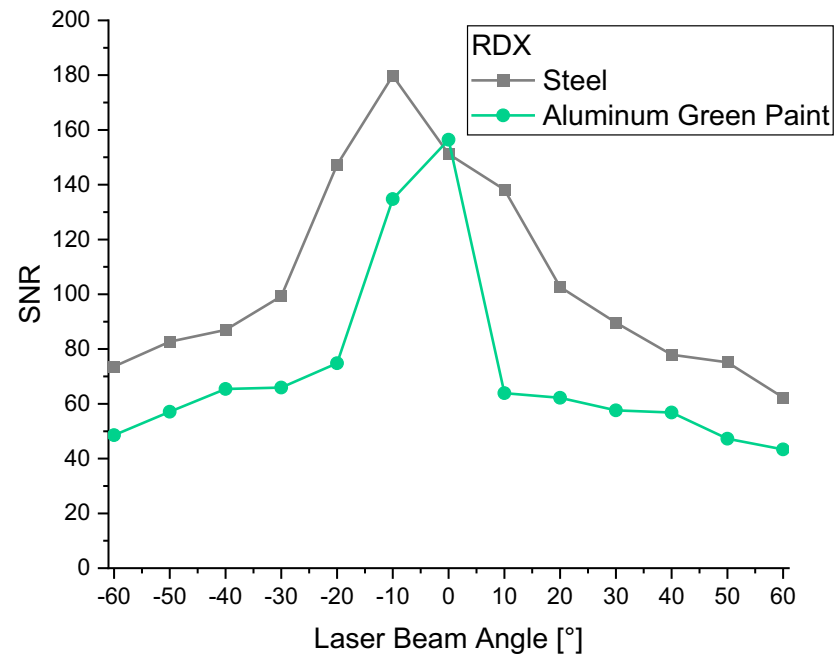

(b)

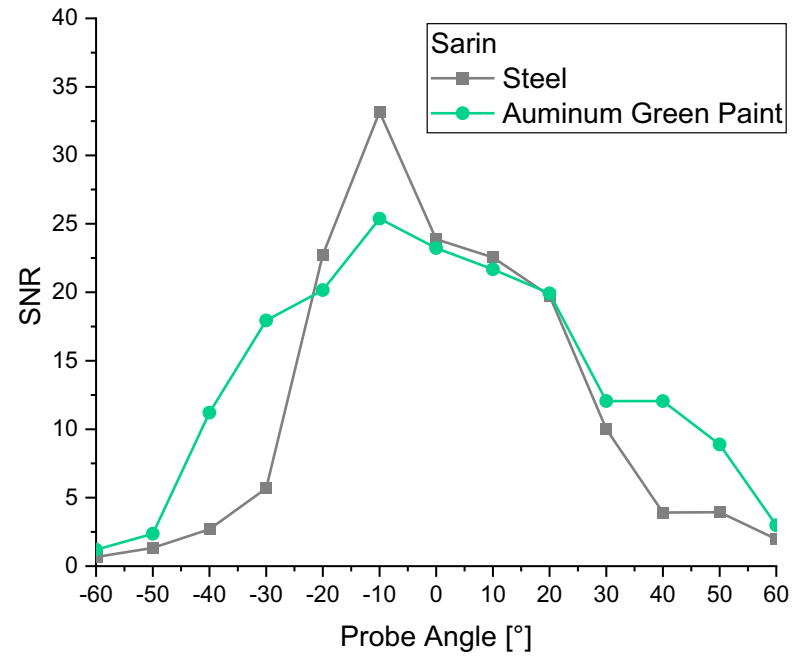

Fig. 18 Incident laser beam angle dependency for a RDX and b Sarin (extrapolated from GLP). Measurements on steel (gray line) and green pain aluminum (green line)

backgrounds. It can be noticed that the SNR of Sarin is smaller than RDX: this could be explained by the fact that the SNR was calculated on the strongest peak of Sarin out of a GLP spectrum acquired. It is speculating that a pure Sarin would give a stronger SNR. This probably would also explain why the signal recorded after $\pm 40^{\circ}$ is lying below the LoD. However, comparing to other laser-based techniques such mid-infrared [1], having a capability to span from at $+30^{\circ}$ to $-30^{\circ}$ and being able to always detect the signal is a considerable advantage.

\subsection{Incident laser beam diameter influence}

A series of tests were performed to investigate how the size of the incident laser beam can affect the detection of hazardous substances. Again, two different chemicals were tested: RDX as representative of the explosives and GLP (simulant of Sarin) as representative of warfare agent simulants. An additional variable was added to the test: two different backgrounds were considered. A stainless steel plate was used as a clean non-interference surface as used as an ideal reference background; the green paint aluminum plate was chosen since it provided fluorescence interference. For the latter background it is important to check how much the laser beam size affects the strength of the fluorescence, hence creating a disturbance to detect the Raman signal. In fact, since the chemical compound (both RDX and GLP) was kept to a constant size $(0.5 \mathrm{~cm}$ diameter), the excitation laser beam was varied in size: the bigger is the diameter, the higher is the percentage of probed background compared to the chemical onto it. All the tests were performed with the 355-nm excitation laser beam kept constant energy density of $10.19 \mathrm{~mJ} / \mathrm{cm}^{2}$ for the entire campaign; the round laser beam was changed in size to have $0.25,0.50,1.00$ and $2.00 \mathrm{~cm}$ diameter, respectively. These sizes correspond to $100 \%$ (laser area smaller than sample size), $100 \%$ (laser area equal to sample size), $25 \%$ and $6 \%$ of the filling factor (ratio area of chemicals/area of background). Figure 19 shows the results for RDX and Sarin (extrapolated by GLP), respectively.

In both figures it can be noticed that the behavior of all the curves is very similar: after an initial value obtained with the laser beam smaller than the sample, the peak of the signal was reached when the filling factor was $100 \%$. This peak was expected since the entire hazardous material was generating signal without or minimal (due to imperfection of the sample) interference from the background. In fact, increasing the laser beam led to a decrease of the signal: although the sample was fully illuminated by the excitation laser beam, a considerable area of the beam itself was occupied by the background. Hence, interference was created that increased the background noise, and consequently the SNR. This interference is even more evident when the fluorescing background (green painted aluminum): the SNR obtained is usually half or less than the corresponding value obtained on stainless steel (non-fluorescing).

A further analysis can be performed: considering just the last three measurement, a linear fit can be calculated to estimate which is the maximum diameter which allows a SNR above the LoD. This information can be very useful to maximize the area of interrogation and minimize the acquisition time in the casa of a big surface scan. For this calculation the data from the green painted aluminum background were considered since in a real scenario unknown surfaces (fluorescing and non-fluorescing) are found. Under these uncertainties, the consideration of the worst case provided confidence about successful results in more favorable cases. Applying the mathematical fit to the green curve in Fig. 19a, it is possible to increase the excitation beam size up to $9.00 \mathrm{~cm}$ diameter $(0.30 \%$ filling factor) for RDX. Considering that the average acquisition time for RDX is $5 \mathrm{~s}$, it is estimated that 13 min is required to have a full scan of $1.00 \mathrm{~m}^{2}$ surface. While applying the mathematical fit to the green curve on Fig. 19b, it is possible to increase the excitation beam size up to $6.00 \mathrm{~cm}$ diameter ( $0.67 \%$ filling factor) for Sarin (extrapolated from GLP). Considering that the average 

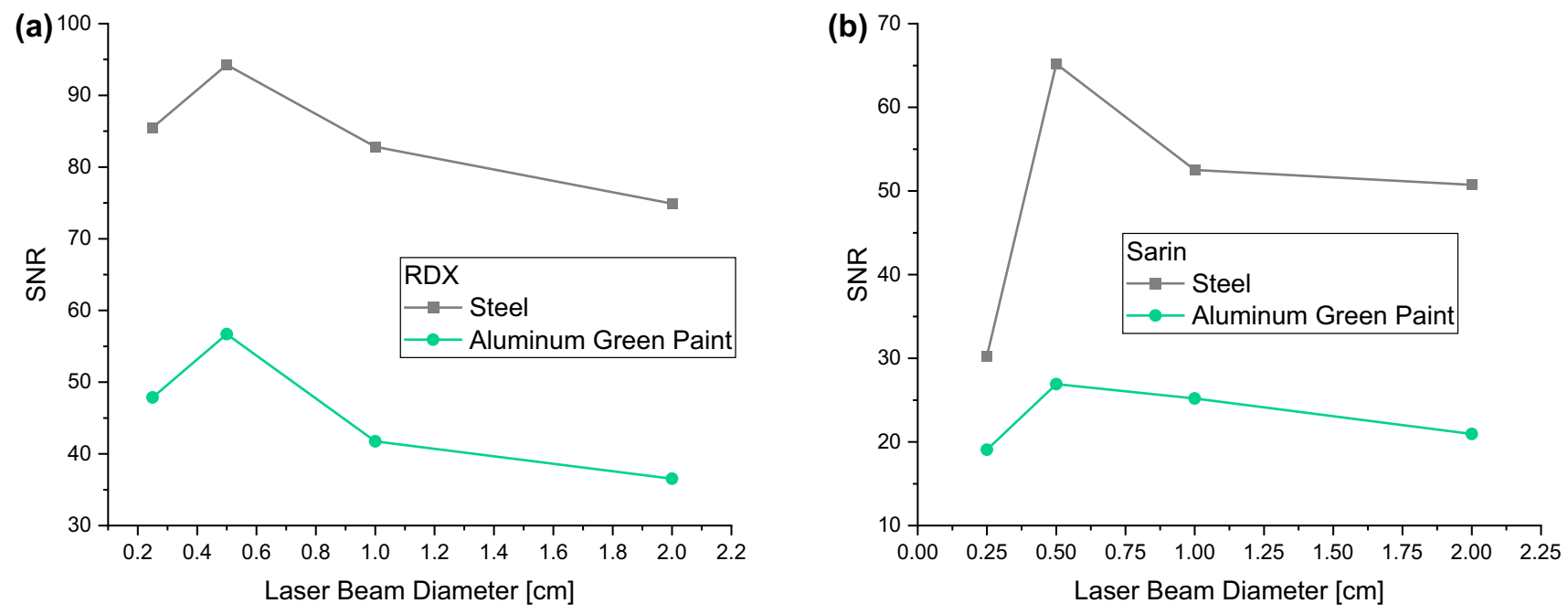

Fig. 19 Incident laser beam diameter influence for $\mathbf{a}$ RDX and $\mathbf{b}$ Sarin (extrapolated from GLP). Measurements on steel (gray line) and green paint aluminum (green line)
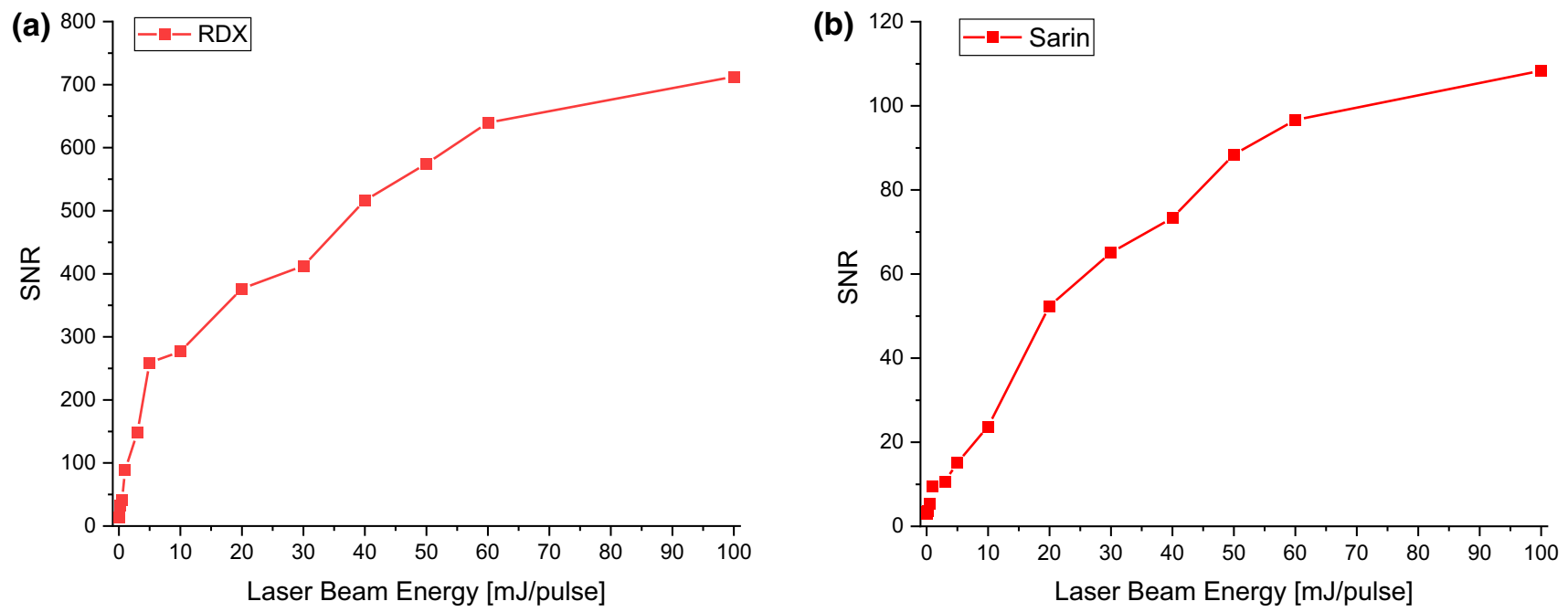

Fig. 20 Incident laser beam energy influence for a RDX and $\mathbf{b}$ Sarin (extrapolated from GLP)

acquisition time for Sarin is $2 \mathrm{~s}$, it is estimated that $12 \mathrm{~min}$ is required to have a full scan of $1.00 \mathrm{~m}^{2}$ surface. It must be reminded that all these numbers are valid for a constant energy density of $10.19 \mathrm{~mJ} / \mathrm{cm}^{2}$.

\subsection{Incident laser beam energy influence}

The last series of tests were performed to investigate how the energy of the incident laser beam can affect the detection of hazardous substances. Again, two different chemicals were tested: RDX as representative of the explosives and GLP (simulant of Sarin) as representative of warfare agent simulants. All the tests were performed using self-made samples: small amount (<1 mg) of RDX and GLP were compacted on both surfaces to produce a round shape of $0.5 \mathrm{~cm}$ diameter. The amount of compounds were chosen such that the Raman signal was strong enough to be detected at any energy change and decoupled from the concentration. In this case, the SNR was expected to be higher than the previous measurements, since the interest was focused only on the energy change dependency. For this study a stainless steel plate was used as a background. The 355-nm excitation laser beam was kept constant for the all tests: the round laser beam had a size of $1.0 \mathrm{~cm}$ diameter at $20 \mathrm{~Hz}$ pulse rate. The laser energy was changed from 0.05 to $100 \mathrm{~mJ} /$ pulse. The energy was measured with a power meter in front of the sample before each test. Figure 20 shows the results for RDX and Sarin (extrapolated by GLP, respectively). It is evident that increasing the energy led to an increase of the signal.

The behavior of both plots is logarithmic, and both figures show a plateau in the range of $100 \mathrm{~mJ} / \mathrm{pulse}$. This indicates that a further increase of the exciting laser energy will not raise the SNR significantly. 
It is important to notice that even the highest laser energy tested did not ignite or detonated RDX. This supports the initial statement of this study that the short-pulse laser provided enough relaxation time for the molecules to return to their ground state before being re-excited by the following pulse. A further consideration: the minimum energy required to have SNR above the LoD was $0.1 \mathrm{~mJ} /$ pulse. This value was obtained under ideal conditions, but it proves that the measurements are possible keeping the excitation laser energy below the eye-safe level ( $<3 \mathrm{~m} \mathrm{~J} / \mathrm{cm}^{2}$, MPEs) [27], enabling the possibility to use a Raman-based device in public/crowded places.

\section{Conclusions}

A remote detection Raman-based optical system was successfully setup and optimized to detect traces of selective explosives (RDX and TNT) and warfare agent simulants (GLP, TBP, TEP and TPP) at $50 \mathrm{~cm}$ distance on realistic backgrounds. Initial measurements performed on standard NATO-SET-237 samples demonstrated the capability of the system to be highly selective (the Raman signature of each chemical was well captured) and highly sensitive. The minimum detected values are found to be $650 \mathrm{ng}$ for RDX and $820 \mathrm{ng}$ for TNT. Mathematical interpolations proved that the minimum detectable amount can be theoretically detected below $1.0 \mu \mathrm{g}$ for other backgrounds too. Explosives and warfare agent simulants were successfully tested on realistic backgrounds as well. Eighteen different materials were used as backgrounds and divided in five categories: construction materials, metals, natural materials, transparent materials and plastic. Chemicals were spread onto them to produce uneven and uniform samples to simulate a realistic warzone scenario. A laser microscope analysis performed after the measurements was employed to determine the exact quantity probed. Results demonstrated that the Raman spectroscopy provided high selectivity and high sensitivity also over non-ideal samples. Despite interference due to non-perfect scattering (due to uneven and rough surfaces) and interferences from the room light and native fluorescence (both from chemicals and from backgrounds), the system was always able to detect RDX below $10 \mu \mathrm{g}$ and TNT below $26 \mu \mathrm{g}$ while warfare agent simulants below $1.8 \mathrm{mg}$. It must be considered that while explosive amounts were calculated exactly (penetration depth was known from literature), the warfare agents were overestimated: the scattered volume was calculated using the measured thickness by the laser microscope. Mathematical interpolations were used as well to identify the theoretical minimum detectable quantity for each background. It was demonstrated that it was possible to detect explosives in the ng range also on realistic backgrounds, while warfare agents were mostly detected below the toxicology limits $(500 \mu \mathrm{g})$. Further tests were performed to test the angle of the incident laser beam dependency, the laser beam diameter influence and the laser beam energy influence. It was demonstrated that the peak of the Raman signal was found to be within $\mathrm{a} \pm 30^{\circ}$ off from the optimal configuration. This result provided a good flexibility of the system guaranteeing successful measurements even when it is outside the optimal configuration. Also, the second test demonstrated that increasing the laser beam size leads to a faster detection. However, the fill factor (ratio area of the chemical/area of the background) limited the maximum area of the incident laser beam. It was calculated that for the given test conditions ( $355 \mathrm{~nm}$ excitation, wavelength, $10.19 \mathrm{~mJ} / \mathrm{cm}^{2}$ energy density at $20 \mathrm{~Hz}$ repletion rate) it is possible to measure up to $0.30 \%$ filling factor for RDX and $0.67 \%$ filling factor for Sarin (extrapolated from GLP). Therefore, it was estimated that it is required between 12 and 13 min to scan completely a $1 \mathrm{~m}^{2}$ surface. Laser energy tests demonstrated the Raman signal increased logarithmically with the increase of the energy and that neither ignition nor detonation of the samples occurred up to $100 \mathrm{~mJ} /$ pulse. In addition, it was proved the possibility to detect explosives and warfare agents with laser energies below the eye-safe threshold. Since the optical setup was optimized, a further development would be miniaturizing the optical system and the electronics in order to produce a small portable device. Further tests would also be needed to build libraries for identification software that can provide a live response for a possible threat.

Open Access This article is licensed under a Creative Commons Attribution 4.0 International License, which permits use, sharing, adaptation, distribution and reproduction in any medium or format, as long as you give appropriate credit to the original author(s) and the source, provide a link to the Creative Commons licence, and indicate if changes were made. The images or other third party material in this article are included in the article's Creative Commons licence, unless indicated otherwise in a credit line to the material. If material is not included in the article's Creative Commons licence and your intended use is not permitted by statutory regulation or exceeds the permitted use, you will need to obtain permission directly from the copyright holder. To view a copy of this licence, visit http://creativecommons.org/licenses/by/4.0/.

\section{References}

1. P. M Pellegrino., E. L.Holthoff, M. E.Farrell, [Laser-based optical detection of explosives], CRC Press, New York (2015)

2. P. Zang, Y. Tang, A. Shi, L. Bao, Y. Shen, R. Shen, Y. Ye, Recent developments in spectroscopic techniques for the detection of explosives. Materials 11(8), 1364 (2018). https://doi.org/10.3390/ma11081364

3. K.L. Gares, K.T. Hufziger, S.V. Bykov, S.A. Asher, Review of explosive detection methodologies and the emergence of stand-off deep UV resonance raman. J. Raman Spectrosc. 47, 124-141 (2016). https://doi.org/10.1002/jrs.4868

4. J.K. Chung, S.G. Cho, Standoff Raman Spectroscopic detection of Explosive Molecules. Bull.Korean Chem. Soc. (2013). https://doi.org/10.5012/bkcs. 2013.34.6.1668

5. E.C.A. Gallo, L.M.L. Cantu, F. Duschek, Remote Raman Spectroscopy of Explosive Precursors. Opt. Eng. (2021). https://doi.org/10.1117/1.OE.60.8. 084108

6. F. Kullander, H. Lunden, P. Wästerby, L. Landström, Ultraviolet raman scattering from V-Agents. Proc. of SPIE (2017). https://doi.org/10.1117/12. 2262123 
7. E.C.A. Gallo, L.M.L. Cantu, F. Duschek, RDX remote raman detection on NATO-SET-237 samples. Eur. Phys. J. Plus 136, 401 (2021). https://doi.org/ 10.1140/epjp/s13360-021-01336-9

8. Perhydro-1,3,5-trinitro-1,3,5-triazina, ECA (European Chemical Agency), https://echa.europa.eu/brief-profile/-/briefprofile/100.004.092

9. 2,4,6-trinitrotoluene, European Chemical Agency, https://echa.europa.eu/registration-dossier/-/registered-dossier/16165/1

10. Glyphosate, European Chemical Agency, https://echa.europa.eu/hot-topics/glyphosate

11. Triphenyl phosphate, European Chemical Agency, https://echa.europa.eu/brief-profile/-/briefprofile/100.003.739

12. B.C. Singer, A.T. Hodgson, H. Destaillatis, T. Hotchi, K.L. Revzan, R.G. Sexto, Indoor Sorption of Surrogates for Sarin and Related Nerve Agents. Environ. Sci. Technol. 39(9), 3203-3214 (2005). https://doi.org/10.1021/es049144u

13. [NATO SET-237 Printed Standards for Standoff Detection], Document AC/323-D(2018)0001 (2018)

14. F. Schnürer, C. Ulrich, S. Müller, W. Schweikert, Printed explosives standards for the evaluation of stand-off optical systems. Proc. of SPIE (2018). https://doi.org/10.1117/12.2325305

15. H. Yu, D.A. DeTata, S.W. Lewis, N.N. Daeid, The stability of TNT, RDX and PETN in simulated post-explosion soils: implications of sample preparation for analysis. Talanta 164, 716-726 (2017). https://doi.org/10.1016/j.talanta.2016.07.001

16. W. S. Rasband, ImageJ, US National Institute of Health, Bethesda, MD, USA (1997-2021) http://rsb.info.nih.gov/ij

17. W. Fountain III., S.D. Christensen, R.P. Moon, J.A. Guichetau, E.D. Emmons, Recent advances and remaining challenges for the spectroscopic detection of explosive threats. Appl. Spectrosc. 68, 795-811 (2014). https://doi.org/10.1366/14-07560

18. L. Nagli, M. Gaft, Y. Fleger, M. Rosenbluh, Absolute raman cross-section of some explosives: trend to UV. Opt. Mater. 30, 1747-1754 (2008). https:// doi.org/10.1016/j.optmat.2007.11.019

19. M. Amin, P. Wen, W.D. Herzog, R.R. Kunz, Optimization of ultraviolet raman spectroscopy for trace explosive checkpoint screening. Anal. Bioanal. Chem. 412, 4495-4504 (2020). https://doi.org/10.1007/s00216-020-02725-2

20. V.P. Shrivastava Guptha, Methods for the determination of limit of detection and limit of quantification of the analytical methods". Chron. Young Sci. 2(1), 21-25 (2011). https://doi.org/10.4103/2229-5186.79345

21. D.D. Tuschel, A.V. Mikhonin, B.E. Lemoff, S.A. Asher, Deep ultraviolet resonance raman excitation enables explosives detection. Appl. Spectrosc. 64(4), 425-432 (2010). https://doi.org/10.1366/000370210791114194

22. Z. Dreger, M.Y. Gupta, High Pressure raman spectroscopy of single crystals of hexahydro-1,3,5-trinitro-1,3,5-triazine (RDX). J. Phys. Chem. B 111, 3893-3903 (2007). https://doi.org/10.1021/jp0681092

23. C. Eckbreth, Laser diagnostics for combustion temperature and species, 2nd edition (Taylor \& Francis, New York NY, 2002)

24. H. Bhakhoa, L. Rhyman, P. Ramasami, Theoretical study of the molecular aspect of the suspected novichok agent A234 of the Skripal Poisoning. Royal Soc. Open Sci. (2019). https://doi.org/10.1098/rsos.181831

25. S. Farquharson, A. Gift, P. Maksymiuk, F. Inscore, Surface-Enhanced Raman Spectra of VX and its hydrolysis products. Appl. Spectrosc. 59, 654-659 (2005)

26. National Research Council, Acute exposure guideline levels for selective airborne chemicals, vol. 3 (The National Academy Press, Washington DC, 2003)

27. S. Almaviva, R. Chirico, M. Nuvoli, A. Palucci, F. Schnürer, W. Schweikert, A new eye-safe UV raman spectrometer for the remote detection of energetic materials in fingerprints concentrations: characterization by PCA and ROC analyzes. Elsevier Talanta 144, 420-426 (2015). https://doi.org/10.1016/j. talanta.2015.06.075 